Dept. of Math. University of Oslo

Pure Mathematics No. 19

ISSN 0806-2439 JUNE 2004

\title{
UNIQUENESS OF ENTROPY SOLUTIONS FOR \\ DOUBLY NONLINEAR ANISOTROPIC DEGENERATE PARABOLIC EQUATIONS
}

\author{
MOSTAFA BENDAHMANE AND KENNETH H. KARLSEN
}

\begin{abstract}
We consider doubly nonlinear anisotropic degenerate parabolic equations, supplemented with an initial condition and a homogeneous Dirichlet boundary condition. We introduce a notion of entropy solution and prove that the entropy solution is uniquely determined by its data.
\end{abstract}

\section{INTRODUCTION}

We introduce a notion of entropy solution and prove a uniqueness result for doubly nonlinear anisotropic degenerate parabolic equations, supplemented with an initial condition and a homogeneous Dirichlet boundary condition.

The problems under consideration take the form

$$
\left\{\begin{array}{l}
\partial_{t} u+\sum_{i=1}^{d} \partial_{x_{i}} f_{i}(u)=\sum_{i=1}^{d} \partial_{x_{i}}\left(\left|\partial_{x_{i}} A_{i}(u)\right|^{p_{i}-2} \partial_{x_{i}} A_{i}(u)\right) \text { in } Q_{T}, \\
\left.u\right|_{t=0}=u_{0} \text { in } \Omega \text { and } u=0 \text { on }(0, T) \times \partial \Omega,
\end{array}\right.
$$

where $u(t, x): Q_{T} \rightarrow \mathbf{R}$ is the unknown function that is sought, $Q_{T}=(0, T) \times \Omega$, $T>0$ is a fixed time, $\Omega \subset \mathbf{R}^{d}$ is a bounded domain with smooth boundary $\partial \Omega$, and $p_{i}>1$ for $i=1, \ldots, d$. The initial function $u_{0}: \Omega \rightarrow \mathbf{R}$ is assumed to be bounded.

The functions $A_{1}, \ldots, A_{d}: \mathbf{R} \rightarrow \mathbf{R}^{d}$ satisfy

(1.2) $\quad A_{i} \in \operatorname{Lip}_{\text {loc }}(\mathbf{R})$ and $A_{i}(\cdot)$ is nondecreasing with $A_{i}(0)=0, i=1, \ldots, d$, while the convective flux function $f=\left(f_{1}, \ldots, f_{d}\right): \mathbf{R} \rightarrow \mathbf{R}^{d}$ satisfies

$$
f(u) \in \operatorname{Lip}_{\text {loc }}\left(\mathbf{R}^{d} ; \mathbf{R}^{d}\right) \text { and } f(0)=0 .
$$

Let us also state a closely related problem, namely the following one containing an "isotropic" second order operator:

$$
\left\{\begin{array}{l}
\partial_{t} u+\operatorname{div} f(u)=\operatorname{div}\left(|\nabla A(u)|^{p-2} \nabla A(u)\right), \quad \text { in } Q_{T}, \\
\left.u\right|_{t=0}=u_{0} \text { in } \Omega \text { and } u=0 \text { on }(0, T) \times \partial \Omega
\end{array}\right.
$$

where $p>1$ and $A(\cdot)$ is a scalar nondecreasing Lipschitz function with $A(0)=0$. Note that even when $p_{i}=p \neq 2$ and $A_{i} \equiv A$ for all $i$, the anisotropic problem (1.1) does not coincide with (1.4) (but it does when $p=2$ ).

Since the graph of $A_{i}(\cdot)$ can be flat, solutions to (1.1) will in general develop discontinuities, even when the initial function $u_{0}$ is smooth. Hence it becomes necessary to interpret (1.1) in the weak sense. Moreover, it is well known from the theory of conservation laws that discontinuous weak solutions are not uniquely

Date: June 7, 2004

Key words and phrases. degenerate parabolic equation, entropy solution, uniqueness.

This work was supported by the BeMatA program of the Research Council of Norway and the European network HYKE, funded by the EC as contract HPRN-CT-2002-00282. 
determined by their data and consequently an additional selection criterion, a socalled entropy condition, is needed to single out a unique weak solution.

Indeed, the conservation law

$$
\partial_{t} u+\operatorname{div} f(u)=0
$$

is a special case of (1.1). A rather complete $L^{\infty}$ entropy solution theory for the Cauchy problem for scalar conservation laws was developed by Kružkov [31] and Vol'pert [44]. A detailed exposition of Kružkov's theory can be found in, e.g., [33]. We refer to $[3,37,33,46]$ for a corresponding theory for the Dirichlet boundary value problem.

Many other partial differential equations (usually possessing more regular solutions) are also special cases of (1.1) and (1.4). Let us mention the heat and porous medium equations

$$
\partial_{t} u=\Delta u, \quad \partial_{t} u=\Delta u^{m}, \quad m>1,
$$

and more generally degenerate convection-diffusion equations of the type

$$
\partial_{t} u+\sum_{i=1}^{d} \partial_{x_{i}} f_{i}(u)=\sum_{i=1}^{d} \partial_{x_{i} x_{i}}^{2} A_{i}(u) \quad\left(A_{i}\right. \text { satisfies (1.2)). }
$$

Degenerate parabolic equations like (1.5) occur in theories of flow in porous media (see discussion and references in [21]) and sedimentation-consolidation processes [11].

Another famous representative of the class of equations that is considered herein is the $p$-Laplace equation

$$
\partial_{t} u=\operatorname{div}\left(|\nabla u|^{p-2} \nabla u\right), \quad p>1,
$$

which arises in the theory of non-Newtonian filtration. Also well known is the doubly nonlinear polytropic filtration equation

$$
\partial_{t} u=\operatorname{div}\left(\left|\nabla\left(|u|^{m-1} u\right)\right|^{p-2} \nabla\left(|u|^{m-1} u\right)\right), \quad m, p>1 .
$$

Degenerate parabolic equations of the type just mentioned have been intensively studied in recent decades, see the books $[19,48]$ and the references cited therein (some recent regularity results for (1.6) can be found in [20]). A related class of equations consists of the so-called elliptic-parabolic equations

$$
\partial_{t} b(u)=\operatorname{div} a(u, \nabla u)
$$

where $b: \mathbf{R} \rightarrow \mathbf{R}$ is a continuous nondecreasing function ( $b$ can be flat) and $a(r, \xi): \mathbf{R} \times \mathbf{R}^{N} \rightarrow \mathbf{R}^{N}$ is continuous, monotone in $\xi$ and satisfies a growth condition of the type $|a(r, \xi)| \leq C(r)\left(1+|\xi|^{p-1}\right), p>1$. We refer to $[1,7,38,14]$ and the references cited therein for more information on elliptic-parabolic equations.

In most of the situations mentioned above, solutions possessing some type of Sobolev regularity in the spatial variable are sought. The problems we have in mind will in general possess discontinuous solutions. Consequently, it becomes more challenging to devise reasonable solution concepts and to prove uniqueness/stability results. In recent years the isotropic problem (1.4) with $p=2$ has caught a great deal of attention. A first study of entropy solutions for such equations is due to Vol'pert and Hudjaev [45]. For one-dimensional equations, some general uniqueness results have been proved by $\mathrm{Wu}$ and Yin [47] (see also the book [48]) and Bénilan and Touré [6]. In the multi-dimensional context a general uniqueness result is due to Carrillo [13, 12]. He was the first to successfully implement Kružkov's doubling of the variables device [31] for second order equations. He also developed a powerful method for handling a homogeneous Dirichlet boundary condition in degenerate problems. Various extensions of his result can be found in [10, 25, 28, 30, 34, 35, 40], 
see also [15] for a different approach and [41] for a uniqueness proof for piecewise smooth weak solutions. Among the works cited, we mention that $[34,35]$ analyze the problem with a non-homogeneous Dirichlet boundary condition. Explicit estimates for continuous dependence on the nonlinearities were proved in [18], see also [30, 24]. Several recent studies concerned with the convergence of numerical schemes can be found in $[2,9,21,23,25,26,29,36]$.

The anisotropic degenerate parabolic equation (which generalizes (1.5))

$$
\partial_{t} u+\operatorname{div} f(u, t, x)=\operatorname{div}(a(u) \nabla u), \quad a(u)=\sigma(u) \sigma(u)^{\top} \geq 0,
$$

where $\sigma(u) \in L_{\text {loc }}^{\infty}\left(\mathbf{R} ; \mathbf{R}^{d \times K}\right), 1 \leq K \leq d$, was treated very recently by Chen and Perthame [17] (for the Cauchy problem). They introduced notions of entropy and kinetic solutions and proved existence and uniqueness results for such solutions. One key point in their notions of solution is to explicitly include the parabolic dissipation measure in the entropy inequality. This is different from Carrillo's approach, which attempts to recover a particular form of the parabolic dissipation measure from the Kružkov entropy inequality (this seems to work only for isotropic equations). Uniqueness was proved in [17] using a kinetic formulation and regularization by convolution. An alternative theory for (1.7) based on Kružkov's doubling of the variables device was developed in [4]. The generality in [17, 4] allows for pure $L^{1}$ initial data $u_{0}$, while herein we consider only the case $u_{0} \in L^{\infty}$. Within the kinetic framework, explicit continuous dependence and error estimates for entropy solutions of (1.7) were obtained in [16]. In [39], a notion of dissipative solution for (1.7) and its relation to the notion of entropy solution is studied. Moreover, a convergence proof is given for certain relaxation approximations.

Despite recent efforts, problems (1.1) and (1.4) are still in general very poorly investigated. Let $A, B: \mathbf{R} \rightarrow \mathbf{R}$ be nondecreasing Lipschitz functions such that $B( \pm \infty)= \pm \infty$. Then some time ago Yin [49] studied the one-dimensional problem

$$
\partial_{t} u+\partial_{x} f(u)=\partial_{x} B\left(\partial_{x} A(u)\right) \text { on }(0, T) \times(0,1),\left.u\right|_{t=0}=u_{0},\left.u\right|_{x=0,1}=0
$$

in a framework of discontinuous entropy solutions having bounded variation (in both $t$ and $x$ ). He proved existence as well as uniqueness and stability results. The Cauchy problem was studied in [22] via a finite difference approach. When $d=1$ and $\Omega=(0,1),(1.4)$ is obtained from Yin's problem by choosing $B(\xi)=|\xi|^{p-2} \xi$.

In the multi-dimensional context, it seems that the only results up to now are those of Igbida and Urbano [27] (see also previous work by Urbano [42, 43]). They prove existence and uniqueness results for weak solutions of the isotropic problem (1.4) under the additional structure condition

$$
f(u)=F(A(u)), \quad \text { for some Lipschitz function } F: \mathbf{R} \rightarrow \mathbf{R}^{d}, F(0)=0 .
$$

Uniqueness of the weak solution is elegantly obtained by verifying that any weak solution is also an entropy solution and then using the doubling of the variables approach developed by Carrillo [13] for entropy solutions.

The aim of this paper is to provide a solution theory that avoids any structure condition like (1.8) and more importantly is able to encompass the anisotropic problem (1.1). As already indicated above, Carrillo's approach is a good one when the second order differential operator is isotropic. However, it is not applicable to an anisotropic problem like (1.1). Instead we shall rely on the Kružkov approach developed in [4]. The paper [4] dealt with the Cauchy problem for (1.7) and $L^{1}$ initial data $u_{0}$. Herein we consider an initial-boundary value problem with $L^{\infty}$ initial data. To incorporate the homogeneous Dirichlet boundary condition we shall borrow some ideas from [13]. Even for (1.5) our results are new, as only the Cauchy problem was treated in $[17,4]$. 
The remaining part of this paper is organized as follows: We use Section 2 to introduce and discuss our notion of entropy solution for (1.1). We then state and prove our main uniqueness theorem for these entropy solutions in Section 3. Except for Remark 2.4, a complete proof of the existence of an entropy solution will be postponed to our forthcoming paper [5]. In that paper we also develop a theory based on a notion of renormalized entropy solution that allows for possibly unbounded (pure $L^{1}$ ) initial data $u_{0}$. A similar theory for (1.7) can found in [4].

Finally, we mention that future work will be devoted to the convergence analysis of numerical schemes for (1.1). We refer to [22] for some work in that direction in the one-dimensional context.

\section{Definition OF ENTROPY SOLUTION}

For $1 \leq i \leq d$, we set

$$
\zeta_{i}(u)=\int_{0}^{u}\left(A_{i}^{\prime}(\xi)\right)^{\frac{p_{i}-1}{p_{i}}} d \xi, \quad \zeta(u)=\left(\zeta_{1}(u), \ldots, \zeta_{d}(u)\right),
$$

and for any $\psi \in L_{\mathrm{loc}}^{\infty}(\mathbf{R})$

$$
\zeta_{i}^{\psi}(u)=\int_{0}^{u} \psi(\xi)\left(A_{i}^{\prime}(\xi)\right)^{\frac{p_{i}-1}{p_{i}}} d \xi, \quad \zeta^{\psi}(u)=\left(\zeta_{1}^{\psi}(u), \ldots, \zeta_{d}^{\psi}(u)\right)
$$

Definition 2.1. We call $(\eta, q)$, with $\eta: \mathbf{R} \rightarrow \mathbf{R}$ and $q=\left(q_{1}, \ldots, q_{d}\right): \mathbf{R} \rightarrow \mathbf{R}^{d}$, an entropy-entropy flux pair if

$$
\eta \in C^{2}(\mathbf{R}), \quad \eta^{\prime \prime} \geq 0, \quad q^{\prime}=\eta^{\prime} f^{\prime}
$$

If, in addition,

$$
\eta(0)=0, \quad \eta^{\prime}(0)=0, \quad q(0)=0,
$$

we call $(\eta, q)$ a boundary entropy-entropy flux pair.

Remark 2.1. The terminology "boundary entropy-entropy flux pair" is borrowed from Otto's work on scalar conservation laws and boundary conditions, see [33, 37].

We now introduce an appropriate notion of entropy solution:

Definition 2.2 (entropy solution). An entropy solution of (1.1) is a measurable function $u: Q_{T} \rightarrow \mathbf{R}$ satisfying the following conditions:

(D.1) (regularity) $u \in L^{\infty}\left(Q_{T}\right)$ and

$$
\partial_{x_{i}} \zeta_{i}(u) \in L^{p_{i}}\left(Q_{T}\right), \quad i=1, \ldots, d
$$

(D.2) (interior entropy condition) For any entropy-entropy flux pair $(\eta, q)$,

$$
\begin{aligned}
\partial_{t} \eta(u) & +\sum_{i=1}^{d} \partial_{x_{i}} q_{i}(u)-\sum_{i=1}^{d} \partial_{x_{i}}\left(\eta^{\prime}(u)\left|\partial_{x_{i}} A_{i}(u)\right|^{p_{i}-2} \partial_{x_{i}} A_{i}(u)\right) \\
& \leq-\sum_{i=1}^{d} \eta^{\prime \prime}(u)\left|\partial_{x_{i}} \zeta_{i}(u)\right|^{p_{i}} \quad \text { in } \mathcal{D}^{\prime}([0, T) \times \Omega)
\end{aligned}
$$


that is, for any $0 \leq \phi \in \mathcal{D}([0, T) \times \Omega)$,

$$
\begin{aligned}
\int_{Q_{T}}\left(\eta(u) \partial_{t} \phi\right. & +\sum_{i=1}^{d} q_{i}(u) \partial_{x_{i}} \phi \\
& \left.-\sum_{i=1}^{d} \eta^{\prime}(u)\left|\partial_{x_{i}} A_{i}(u)\right|^{p_{i}-2} \partial_{x_{i}} A_{i}(u) \partial_{x_{i}} \phi\right) d x d t \\
& +\int_{\Omega} \eta\left(u_{0}\right) \phi(0, x) d x \geq \int_{Q_{T}} \sum_{i=1}^{d} \eta^{\prime \prime}(u)\left|\partial_{x_{i}} \zeta_{i}(u)\right|^{p_{i}} \phi d x d t
\end{aligned}
$$

(D.3) (boundary entropy condition) For any boundary entropy-entropy flux pair $(\eta, q)$ and for any $0 \leq \phi \in \mathcal{D}([0, T) \times \bar{\Omega}),(2.1)$ holds.

(D.4) (boundary condition) For any $\psi \in L_{\mathrm{loc}}^{\infty}(\mathbf{R})$,

$$
\int_{Q_{T}}\left(\operatorname{div} \zeta^{\psi}(u) \phi+\zeta^{\psi}(u) \cdot \nabla \phi\right) d x d t=0, \quad \forall \phi \in \mathcal{D}((0, T) \times \bar{\Omega}) .
$$

Remark 2.2. When $p_{i}=2$ for all $i$, the right-hand side of (2.1) contains the so-called parabolic dissipation measure used in [17, 15, 4]. We term the measure

$$
\mu_{i}^{\eta}:=-\eta^{\prime \prime}(u)\left|\partial_{x_{i}} \zeta_{i}(u)\right|^{p_{i}},
$$

the $p_{i}$-parabolic dissipation measure (associated with the entropy $\eta$ ), $i=1, \ldots, d$.

Remark 2.3. Let us make some comments about the "boundary condition" (D.4). Suppose we know that each component of $\zeta(u)$ belongs to $L^{1}\left(0, T ; W^{1,1}(\Omega)\right)$. Then we can make trace sense to the statement

$$
\left.\zeta(u)\right|_{(0, T) \times \partial \Omega}=0,
$$

which immediately implies (D.4). Except when all the $A_{i}$ 's are the same, the anisotropy in (1.1) prevents us from concluding that each component of $\zeta(u)$ belongs to the space $L^{1}\left(0, T ; W^{1,1}(\Omega)\right)$, so we cannot make trace sense to $(2.2)$. Hence the "integration by parts formula" in (D.4) is introduced into Definition 2.2 as a convenient "weak reformulation" of (2.2) that circumvents the problem of having to know the existence of strong traces. When proving existence of an entropy solution, condition (D.4) must be explicitly checked (see next remark).

Let us also mention that it is possible to obtain (D.4) directly from (D.1) by properly adapting known results for the space

$$
L^{p}\left(0, T ; L^{p}(\operatorname{div} ; \Omega)\right)=\left\{w=\left(w_{1}, \ldots, w_{d}\right) \in\left(L^{p}\left(Q_{T}\right)\right)^{d}: \operatorname{div} w \in L^{p}\left(Q_{T}\right)\right\} .
$$

It is immediate that the vector field $\zeta(u)=\left(\zeta_{1}(u), \ldots, \zeta_{d}(u)\right)$ belongs to this space with $p=\min _{i=1} p_{i}$. This approach, which avoids including the integration by parts formula (D.4) in Definition 2.2, is fully developed in [5].

Remark 2.4. Although the existence proof for (1.1) is postponed to [5], let us indicate its main steps. For $i=1, \ldots, d$, let $A_{\rho, i}$ be a strictly increasing function that converges locally uniformly to $A_{i}$ as $\rho \downarrow 0$. Existence of an entropy solution can then be proved (see [5] for details) by passing to the limit $\rho \downarrow 0$ in a sequence

$$
u_{\rho} \in L^{\infty}\left(Q_{T}\right) \cap C\left(0, T ; L^{2}(\Omega)\right) \cap L^{p}\left(0, T ; W_{0}^{1, p}(\Omega)\right), \quad p:=\min _{i=1, \ldots, d} p_{i}>1,
$$


of solutions to the non-degenerate equations

$$
\partial_{t} u_{\rho}+\sum_{i=1}^{d} \partial_{x_{i}} f_{i}\left(u_{\rho}\right)=\sum_{i=1}^{d} \partial_{x_{i}}\left(\left|\partial_{x_{i}} A_{\rho, i}\left(u_{\rho}\right)\right|^{p_{i}-2} \partial_{x_{i}} A_{\rho, i}\left(u_{\rho}\right)\right) .
$$

The proof of existence of such a sequence is classical, see for example [32].

At least formally, multiplying $(2.3)$ by $\eta^{\prime}\left(u_{\rho}\right), \eta \in C^{2}, q^{\prime}=\eta^{\prime} f^{\prime}$, gives

$$
\begin{aligned}
\partial_{t} \eta\left(u_{\rho}\right) & +\sum_{i=1}^{d} \partial_{x_{i}} q_{i}\left(u_{\rho}\right)-\sum_{i=1}^{d} \partial_{x_{i}}\left(\eta^{\prime}\left(u_{\rho}\right)\left|\partial_{x_{i}} A_{\rho, i}\left(u_{\rho}\right)\right|^{p_{i}-2} \partial_{x_{i}} A_{\rho, i}\left(u_{\rho}\right)\right) \\
& \leq-\sum_{i=1}^{d} \eta^{\prime \prime}\left(u_{\rho}\right)\left|\partial_{x_{i}} \zeta_{\rho, i}\left(u_{\rho}\right)\right|^{p_{i}} \quad \text { in } \mathcal{D}^{\prime}([0, T) \times \Omega),
\end{aligned}
$$

where $\zeta_{\rho, i}(z)=\int_{0}^{z}\left(A_{\rho, i}^{\prime}(\xi)\right)^{\frac{p_{i}-1}{p_{i}}} d \xi$. Choosing $\eta(z)=\frac{1}{2} z^{2}$ and using the boundary condition $\left.u_{\rho}\right|_{(0, T) \times \partial \Omega}=0$, it follows from (2.4) that

$$
\int_{Q_{T}} \sum_{i=1}^{d}\left|\partial_{x_{i}} \zeta_{\rho, i}\left(u_{\rho}\right)\right|^{p_{i}} d x d t \leq \frac{1}{2} \int_{\Omega} u_{0}^{2} d x<\infty .
$$

It is not hard to derive a uniform $B V$ estimate for $u_{\rho}(\cdot, t)$ and prove that $u_{\rho}$ converges to some limit $u \in L^{\infty}\left(Q_{T}\right)$ in $L^{1}\left(Q_{T}\right)$ and $\partial_{x_{i}} \zeta_{\rho, i}\left(u_{\rho}\right) \rightarrow \partial_{x_{i}} \zeta_{i}(u)$ in $L^{p_{i}}\left(Q_{T}\right), i=1, \ldots, d$, at least along subsequences. These convergences, together with a version of Minty's trick, allow us to pass to the limit in (2.4) to obtain that $u$ satisfies (D.2). In this passage to the limit, we also need to employ a standard weak lower semicontinuity result to obtain

$$
\underset{\rho \downarrow 0}{\liminf } \int_{Q_{T}} \sum_{i=1}^{d} \eta^{\prime \prime}\left(u_{\rho}\right)\left|\partial_{x_{i}} \zeta_{\rho, i}\left(u_{\rho}\right)\right|^{p_{i}} \phi d x d t \geq \int_{Q_{T}} \sum_{i=1}^{d} \eta^{\prime \prime}(u)\left|\partial_{x_{i}} \zeta_{i}(u)\right|^{p_{i}} \phi d x d t,
$$

for any nonnegative test function $\phi(t, x)$. Along the same lines, we can also prove that $u$ satisfies (D.3).

It remains to verify (D.4). As $\left.u_{\rho}\right|_{(0, T) \times \partial \Omega}=0$ is understood in the classical trace sense, we clearly have for any $\psi \in L_{\mathrm{loc}}^{\infty}(\mathbf{R})$

$$
\int_{Q_{T}}\left(\operatorname{div} \zeta^{\psi}\left(u_{\rho}\right) \phi+\zeta^{\psi}\left(u_{\rho}\right) \cdot \nabla \phi\right) d x d t=0, \quad \forall \phi \in \mathcal{D}((0, T) \times \bar{\Omega}) .
$$

Exploiting that $u_{\rho} \rightarrow u$ in $L^{1}\left(Q_{T}\right)$ and $\operatorname{div} \zeta^{\psi}\left(u_{\rho}\right) \rightarrow \operatorname{div} \zeta^{\psi}(u)$ in, say, $L^{1}\left(Q_{T}\right)$, we can send $\rho \downarrow 0$ in (2.5) to conclude that the limit $u$ satisfies (D.4).

Remark 2.5. For the uniqueness proof given later, we shall need a particular set of boundary entropy-entropy flux pairs. Let us introduce a $C^{1}$ approximation of

For $\varepsilon>0$, set

$$
\operatorname{sign}^{+}(z)= \begin{cases}0, & z \leq 0 \\ 1, & z>0\end{cases}
$$

$$
\operatorname{sign}_{\varepsilon}^{+}(\xi)= \begin{cases}0, & \xi<0, \\ \sin \left(\frac{\pi}{2 \varepsilon} \xi\right), & 0 \leq \xi \leq \varepsilon \\ 1, & \xi>\varepsilon\end{cases}
$$

and

$$
\eta_{\varepsilon}^{+}(z, c)=\int_{c}^{z} \operatorname{sign}_{\varepsilon}^{+}(\xi-c) d \xi, \quad z, c \in \mathbf{R} .
$$

Then $\eta_{\varepsilon}(\cdot, c)$ belongs to $C^{2}(\mathbf{R})$, is convex, and as $\varepsilon \downarrow 0$

$$
\eta_{\varepsilon}^{+}(z, c) \rightarrow \eta^{+}(z, c):=(z-c)^{+}=\max (z-c, 0) .
$$


Define the entropy flux function

$$
q_{\varepsilon}^{+}(z, c)=\int_{c}^{z} \operatorname{sign}_{\varepsilon}^{+}(\xi-c) f^{\prime}(\xi) d \xi, \quad z, c \in \mathbf{R}
$$

Then as $\varepsilon \downarrow 0$

$$
q_{\varepsilon}^{+}(z, c) \rightarrow q^{+}(z, c):=\operatorname{sign}^{+}(z-c)(f(z)-f(c)) .
$$

Observe that $\left(\eta_{\varepsilon}^{+}(\cdot, c), q_{\varepsilon}^{+}(\cdot, c)\right)_{c \geq 0}$ is a family of boundary entropy-entropy flux pairs. Consequently, using $\eta=\eta_{\varepsilon}^{+}$as an entropy in (2.1) and then sending $\varepsilon \downarrow 0$,

$$
\begin{gathered}
\int_{Q_{T}}\left((u-c)^{+} \partial_{t} \phi+\sum_{i=1}^{d} \operatorname{sign}^{+}(u-c)\left(f_{i}(u)-f_{i}(c)\right) \partial_{x_{i}} \phi\right. \\
\left.-\sum_{i=1}^{d} \operatorname{sign}^{+}(u-c)\left|\partial_{x_{i}} A_{i}(u)\right|^{p_{i}-2} \partial_{x_{i}} A_{i}(u) \partial_{x_{i}} \phi\right) d x d t \\
+\int_{\Omega}\left(u_{0}-c\right)^{+} \phi(0, x) d x \\
\geq \liminf _{\varepsilon \downarrow 0} \int_{Q_{T}} \sum_{i=1}^{d}\left(\operatorname{sign}_{\varepsilon}^{+}\right)^{\prime}(u-c)\left|\partial_{x_{i}} \zeta_{i}(u)\right|^{p_{i}} \phi d x d t,
\end{gathered}
$$

for all pairs $(\phi, c) \in \mathcal{D}([0, T) \times \Omega) \times \mathbf{R}$ and for all pairs $(\phi, c) \in \mathcal{D}([0, T) \times \bar{\Omega}) \times[0, \infty)$ ( $\phi$ is always nonnegative).

Similarly, we introduce the functions

$$
\operatorname{sign}_{\varepsilon}^{-}(\xi)=\left\{\begin{array}{ll}
-1, & \xi<-\varepsilon, \\
\sin \left(\frac{\pi}{2 \varepsilon} \xi\right), & -\varepsilon \leq \xi \leq 0, \\
0, & \xi>0,
\end{array} \quad \eta_{\varepsilon}^{-}(z, c)=\int_{c}^{z} \operatorname{sign}_{\varepsilon}^{-}(\xi-c) d \xi,\right.
$$

which are approximations of

$$
\operatorname{sign}^{-}(z)=\left\{\begin{array}{ll}
-1, & z<0, \\
0, & z \geq 0,
\end{array} \quad \eta^{-}(z, c)=(z-c)^{-}=(-(z-c))^{+} .\right.
$$

For each $z, c \in \mathbf{R}$, define the entropy flux

$$
q_{\varepsilon}^{-}(z, c)=\int_{c}^{z} \operatorname{sign}_{\varepsilon}^{-}(\xi-c) f(\xi) d \xi
$$

Then as $\varepsilon \downarrow 0$

$$
q_{\varepsilon}^{-}(z, c) \rightarrow q^{-}(z, c):=\operatorname{sign}^{-}(z-c)(f(z)-f(c)) .
$$

Consequently, as $\left(\eta_{\varepsilon}^{-}(\cdot, c), q_{\varepsilon}^{-}(\cdot, c)\right)_{c \leq 0}$ is a family of boundary entropy-entropy flux pairs, we can use $\eta=\eta_{\varepsilon}^{-}$as an entropy in (2.1) and then send $\varepsilon \downarrow 0$ to obtain

$$
\begin{gathered}
\int_{Q_{T}}\left((u-c)^{-} \partial_{t} \phi+\sum_{i=1}^{d} \operatorname{sign}^{-}(u-c)\left(f_{i}(u)-f_{i}(c)\right) \partial_{x_{i}} \phi\right. \\
\left.-\sum_{i=1}^{d} \operatorname{sign}^{-}(u-c)\left|\partial_{x_{i}} A_{i}(u)\right|^{p_{i}-2} \partial_{x_{i}} A_{i}(u) \partial_{x_{i}} \phi\right) d x d t \\
\quad+\int_{\Omega}\left(u_{0}-c\right)^{-} \phi(0, x) d x \\
\geq \liminf _{\varepsilon \downarrow 0} \int_{Q_{T}} \sum_{i=1}^{d}\left(\operatorname{sign}_{\varepsilon}^{-}\right)^{\prime}(u-c)\left|\partial_{x_{i}} \zeta_{i}(u)\right|^{p_{i}} \phi d x d t
\end{gathered}
$$


for all $(\phi, c) \in \mathcal{D}([0, T) \times \Omega) \times \mathbf{R}$ and for all $(\phi, c) \in \mathcal{D}([0, T) \times \bar{\Omega}) \times(-\infty, 0](\phi$ is always nonnegative).

Since

$$
\operatorname{sign}^{-}(z-c)=-\operatorname{sign}^{+}(c-z), \quad(z-c)^{-}=(c-z)^{+}, \quad q^{-}(z, c)=q^{+}(c, z),
$$

and

$$
\left(\operatorname{sign}_{\varepsilon}^{-}\right)^{\prime}(z-c)=\left(\operatorname{sign}_{\varepsilon}^{+}\right)^{\prime}(c-z),
$$

we may replace $(2.7)$ by

$$
\begin{gathered}
\int_{Q_{T}}\left((c-u)^{+} \partial_{t} \phi+\sum_{i=1}^{d} \operatorname{sign}^{+}(c-u)\left(f_{i}(c)-f_{i}(u)\right) \partial_{x_{i}} \phi\right. \\
\left.+\sum_{i=1}^{d} \operatorname{sign}^{+}(c-u)\left|\partial_{x_{i}} A_{i}(u)\right|^{p_{i}-2} \partial_{x_{i}} A_{i}(u) \partial_{x_{i}} \phi\right) d x d t \\
+\int_{\Omega}\left(c-u_{0}\right)^{+} \phi(0, x) d x \\
\geq \liminf _{\varepsilon \downarrow 0} \int_{Q_{T}} \sum_{i=1}^{d}\left(\operatorname{sign}_{\varepsilon}^{+}\right)^{\prime}(c-u)\left|\partial_{x_{i}} \zeta_{i}(u)\right|^{p_{i}} \phi d x d t
\end{gathered}
$$

for the same pairs of $(\phi, c)$ as before.

\section{UNIQUENESS OF ENTROPY SOLUTION}

Our main result is the following theorem:

Theorem 3.1. Suppose (1.2) and (1.3) hold. Let $u$ and $v$ be two entropy solutions of (1.1) with initial data $\left.u\right|_{t=0}=u_{0} \in L^{\infty}(\Omega)$ and $\left.v\right|_{t=0}=v_{0} \in L^{\infty}(\Omega)$. Then for a.e. $t \in(0, T)$

$$
\int_{\Omega}(u(t, x)-v(t, x))^{+} d x \leq \int_{\Omega}\left(u_{0}-v_{0}\right)^{+} d x .
$$

Consequently, $\|u(\cdot, t)-v(\cdot, t)\|_{L^{1}(\Omega)} \leq\left\|u_{0}-v_{0}\right\|_{L^{1}(\Omega)}$. If $u_{0} \leq v_{0}$ a.e. in $\Omega$, then $u \leq v$ a.e. in $Q_{T}$. Finally, if $u_{0}=v_{0}$ a.e. in $\Omega$, then $u=v$ a.e. in $Q_{T}$.

Remark 3.1. A definition similar to Definition 2.2 can be used for the simpler isotropic problem (1.4). For this problem we can replace (D.4) by the requirement that $\zeta(u) \in L^{p}\left(0, T ; W_{0}^{1, p}(\Omega)\right)$, where

$$
\zeta(u)=\int_{0}^{u}(A(\xi))^{\frac{p-1}{p}} d \xi
$$

The conclusions of Theorem 3.1 remain valid for (1.4). Since the proofs are similar, we only prove Theorem 3.1 .

We will make repeated use of the following chain rule property.

Lemma 3.1. Let $0 \leq \sigma \in L_{\mathrm{loc}}^{\infty}(\mathbf{R}), \psi \in L_{\mathrm{loc}}^{\infty}(\mathbf{R})$ be given, and define two functions $\beta, \beta^{\psi}: \mathbf{R} \rightarrow \mathbf{R}$ by

$$
\beta(z)=\int_{0}^{z} \sigma(\xi) d \xi, \quad \beta^{\psi}(z)=\int_{0}^{z} \psi(\xi) \sigma(\xi) d \xi .
$$

With $1 \leq p<\infty$ and $1 \leq i \leq d$ fixed, suppose

$$
u \in L^{\infty}(\Omega), \quad \partial_{x_{i}} \beta(u) \in L^{p}(\Omega) .
$$

Then, for any Borel set $B \subset \mathbf{R}$ with $|B|=0$,

$$
\mid\left\{x \in \Omega: u(x) \in B \text { and } \partial_{x_{i}} \beta(u) \neq 0\right\} \mid=0,
$$


and

$$
\partial_{x_{i}} \beta^{\psi}(u(x))=\psi(u(x)) \partial_{x_{i}} \beta(u(x)),
$$

for a.e. $x \in \Omega$ and in $L^{p}(\Omega)$.

Proof. The proof is similar to that in [17]. First of all, the "inverse Sard" property (3.2) is a consequence of [8, Theorem 3.1]. Next suppose that $u$ takes values in a closed interval $I \subset \mathbf{R}$, and introduce the lower semicontinuous function

$$
\beta^{-1}: \mathbf{R} \rightarrow \mathbf{R}, \quad \beta^{-1}(v):=\inf \{\xi \in I \mid \beta(\xi)=v\} .
$$

Denote by $E \subset \mathbf{R}$ the set of $v \in \mathbf{R}$ at which $\beta^{-1}(\cdot)$ is discontinuous. Since $\beta(\cdot)$ is nondecreasing, $E$ is at most countable.

Introduce $v:=\beta(u) \in L^{\infty}(\Omega) \subset L^{1}(\Omega)$, and note that $\partial_{x_{i}} v \in L^{1}(\Omega)$. Let $\Psi(v)=\int_{0}^{v} \psi\left(\beta^{-1}(w)\right) d w$. It is clear that $\Psi \in \operatorname{Lip}_{\text {loc }}(\mathbf{R})$, so that we may use the chain rule (see $[8$, Theorem 3.1$]$ ) to obtain

$$
\partial_{x_{i}} \Psi(v(x))=\Psi^{\prime}(v(x)) \partial_{x_{i}} v(x)=\psi\left(\beta^{-1}(v(x))\right) \partial_{x_{i}} v(x), \quad \text { for a.e. } x \in \mathbf{R}^{d} .
$$

This implies that (3.3) holds a.e. on $\Omega \backslash \mathcal{E}$, where $\mathcal{E}:=\{x \in \Omega: v(x) \in E\}$. On the other hand, since $|E|=0$, we have $\partial_{x_{i}} v=0$ a.e. on $\mathcal{E}$.

As an easy application of Lemma 3.1, we have a chain rule property for entropy solutions.

Lemma 3.2. Let $u$ be an entropy solution to (1.1) and fix $\psi \in L_{\mathrm{loc}}^{\infty}(\mathbf{R})$. We have, for any Borel set $B \subset \mathbf{R}$ with $|B|=0$,

$$
\mid\left\{(t, x) \in Q_{T}: u(t, x) \in B \text { and } \partial_{x_{i}} \zeta(u(t, x)) \neq 0\right\} \mid=0, \quad 1 \leq i \leq d,
$$

and

$$
\partial_{x_{i}} \zeta^{\psi}(u(t, x))=\psi(u(t, x)) \partial_{x_{i}} \zeta(u(t, x)),
$$

for a.e. $(t, x) \in Q_{T}$ and in $L^{p_{i}}\left(Q_{T}\right), i=1, \ldots, d$.

Remark 3.2. Let $u$ be an entropy solution. By the chain rule (3.5)

$$
\partial_{x_{i}} A_{i}(u)=\left(A_{i}^{\prime}(u)\right)^{\frac{1}{p_{i}}} \partial_{x_{i}} \zeta_{i}(u),
$$

so that by $(\mathbf{D} .1)$ there holds $\partial_{x_{i}} A_{i}(u) \in L^{p_{i}}\left(Q_{T}\right), i=1, \ldots, d$. This also implies

$$
\left|\partial_{x_{i}} A_{i}(u)\right|^{p_{i}-2} \partial_{x_{i}} A_{i}(u) \in L^{p_{i}^{\prime}}\left(Q_{T}\right) \cap L^{1}\left(Q_{T}\right), \quad p_{i}^{\prime}=\frac{p_{i}}{p_{i}-1},
$$

for $i=1, \ldots, d$, and thus (2.1) makes sense.

We are now ready to embark on the proof of Theorem 3.1, which roughly speaking is divided into two lemmas (Lemma 3.3 and Lemma 3.5).

Lemma 3.3. Let $u, v$ be entropy solutions of (1.1) with initial data $u_{0}, v_{0} \in L^{\infty}(\Omega)$, respectively. Then, for any nonnegative function $\phi \in \mathcal{D}([0, T) \times \Omega)$,

$$
\begin{gathered}
\int_{Q_{T}}\left((u-v)^{+} \partial_{t} \phi+\sum_{i=1}^{d} \operatorname{sign}^{+}(u-v)\left[f_{i}(u)-f_{i}(v)\right.\right. \\
\left.\quad-\left(\left|\partial_{x_{i}} A_{i}(u)\right|^{p_{i}-2} \partial_{x_{i}} A_{i}(u)-\left|\partial_{x_{i}} A_{i}(v)\right|^{p_{i}-2} \partial_{x_{i}} A_{i}(v)\right)\right] \partial_{x_{i}} \phi d x d t \\
\quad+\int_{\Omega}\left(u_{0}-v_{0}\right)^{+} d x \geq 0 .
\end{gathered}
$$


Proof. The proof borrows ideas from [4]. In what follows, we let $u$ depend on $(t, x) \in Q_{T}$ and $v$ depend on $(s, y) \in Q_{T}$.

Since $u=u(t, x)$ is an entropy solution, (2.6) holds. By choosing $c=v(s, y)$ in (2.6) and then integrating over $(s, y)$, we get

$$
\begin{gathered}
\int_{Q_{T} \times Q_{T}}\left((u-v)^{+} \partial_{t} \phi+\sum_{i=1}^{d} \operatorname{sign}^{+}(u-v)\left(f_{i}(u)-f_{i}(v)\right) \partial_{x_{i}} \phi\right. \\
\left.\quad-\sum_{i=1}^{d} \operatorname{sign}^{+}(u-v)\left|\partial_{x_{i}} A_{i}(u)\right|^{p_{i}-2} \partial_{x_{i}} A_{i}(u) \partial_{x_{i}} \phi\right) d x d t d y d s \\
+\int_{Q_{T} \times(\{0\} \times \Omega)}\left(u_{0}-v\right)^{+} \phi d x d y d s \\
\geq \liminf _{\varepsilon \downarrow 0} \int_{Q_{T}} \sum_{i=1}^{d}\left(\operatorname{sign}_{\varepsilon}^{+}\right)^{\prime}(u-v)\left|\partial_{x_{i}} \zeta_{i}(u)\right|^{p_{i}} \phi d x d t d y d s,
\end{gathered}
$$

for any $0 \leq \phi=\phi(t, x, s, y) \in \mathcal{D}(([0, T) \times \Omega) \times([0, T) \times \Omega))$.

Since $v=v(y, s)$ is an entropy solution, (2.8) holds. By choosing $c=u(t, x)$ in (2.8) and integrating over $(t, x)$, we get

$$
\begin{aligned}
& \int_{Q_{T} \times Q_{T}}\left((u-v)^{+} \partial_{s} \phi+\sum_{i=1}^{d} \operatorname{sign}^{+}(u-v)\left(f_{i}(u)-f_{i}(v)\right) \partial_{y_{i}} \phi\right. \\
& \left.\quad+\sum_{i=1}^{d} \operatorname{sign}^{+}(u-v)\left|\partial_{y_{i}} A_{i}(v)\right|^{p_{i}-2} \partial_{y_{i}} A_{i}(v) \partial_{y_{i}} \phi\right) d x d t d y d s \\
& +\int_{(\{0\} \times \Omega) \times Q_{T}}\left(u-v_{0}\right)^{+} \phi d x d t d y \\
& \geq \liminf _{\varepsilon \downarrow 0} \int_{Q_{T}} \sum_{i=1}^{d}\left(\operatorname{sign}_{\varepsilon}^{+}\right)^{\prime}(u-v)\left|\partial_{y_{i}} \zeta_{i}(v)\right|^{p_{i}} \phi d x d t d y d s,
\end{aligned}
$$

for any $0 \leq \phi=\phi(t, x, s, y) \in \mathcal{D}(([0, T) \times \Omega) \times([0, T) \times \Omega))$.

Adding (3.7) and (3.8) yields

$$
\begin{gathered}
\int_{Q_{T} \times Q_{T}}\left((u-v)^{+}\left(\partial_{t}+\partial_{s}\right) \phi+\sum_{i=1}^{d} \operatorname{sign}^{+}(u-v)\left(f_{i}(u)-f_{i}(v)\right)\left(\partial_{x_{i}}+\partial_{y_{i}}\right) \phi\right. \\
-\sum_{i=1}^{d} \operatorname{sign}^{+}(u-v)\left(\left|\partial_{x_{i}} A_{i}(u)\right|^{p_{i}-2} \partial_{x_{i}} A_{i}(u) \partial_{x_{i}} \phi\right. \\
\left.\left.-\left|\partial_{y_{i}} A_{i}(v)\right|^{p_{i}-2} \partial_{y_{i}} A_{i}(v) \partial_{y_{i}} \phi\right)\right) d x d t d y d s \\
+\int_{Q_{T} \times(\{0\} \times \Omega)}\left(u_{0}-v\right)^{+} \phi d x d y d s+\int_{(\{0\} \times \Omega) \times Q_{T}}\left(u-v_{0}\right)^{+} \phi d x d t d y \\
\geq \liminf _{\varepsilon \downarrow 0} \int_{Q_{T} \times Q_{T}} \sum_{i=1}^{d}\left(\operatorname{sign}_{\varepsilon}^{+}\right)^{\prime}(u-v)\left(\left|\partial_{x_{i}} \zeta_{i}(u)\right|^{p_{i}}+\left|\partial_{y_{i}} \zeta_{i}(v)\right|^{p_{i}}\right) \phi d x d t d y d s .
\end{gathered}
$$

Let us now specify the test function. To this end, let

$$
\omega_{n}: \mathbf{R}^{d} \rightarrow \mathbf{R}, \quad \omega_{l}: \mathbf{R} \rightarrow \mathbf{R}, \quad n, l \geq 1,
$$

be standard mollifier sequences. Then we take our test function to be

$$
\phi_{n, l}(t, x, s, y)=\phi\left(\frac{x+y}{2}, \frac{t+s}{2}\right) \omega_{n}\left(\frac{x-y}{2}\right) \omega_{l}\left(\frac{t-s}{2}\right) \equiv \phi \omega_{n} \omega_{l},
$$


where $0 \leq \phi \in \mathcal{D}([0, T) \times \Omega)$. With this choice, we have the following properties that will be used repeatedly:

$$
\left(\partial_{t}+\partial_{s}\right) \phi_{n, l}=\left(\partial_{t}+\partial_{s}\right) \phi \omega_{n} \omega_{l}
$$

and for $i=1, \ldots, d$

$$
\left(\partial_{x_{i}}+\partial_{y_{i}}\right) \phi_{n, l}=\left(\partial_{x_{i}}+\partial_{y_{i}}\right) \phi \omega_{n} \omega_{l}
$$

For $i=1, \ldots, d$, let us write

$$
\begin{aligned}
\left|\partial_{x_{i}} A_{i}(u)\right|^{p_{i}-2} \partial_{x_{i}} A_{i}(u) \partial_{x_{i}} \phi_{n, l}-\left|\partial_{y_{i}} A_{i}(v)\right|^{p_{i}-2} \partial_{y_{i}} A_{i}(v) \partial_{y_{i}} \phi_{n, l} \\
=\left(\left|\partial_{x_{i}} A_{i}(u)\right|^{p_{i}-2} \partial_{x_{i}} A_{i}(u)-\left|\partial_{y_{i}} A_{i}(v)\right|^{p_{i}-2} \partial_{y_{i}} A_{i}(v)\right)\left(\partial_{x_{i}}+\partial_{y_{i}}\right) \phi_{n, l} \\
\quad-\left|\partial_{x_{i}} A_{i}(u)\right|^{p_{i}-2} \partial_{x_{i}} A_{i}(u) \partial_{y_{i}} \phi_{n, l}+\left|\partial_{y_{i}} A_{i}(v)\right|^{p_{i}-2} \partial_{y_{i}} A_{i}(v) \partial_{x_{i}} \phi_{n, l} .
\end{aligned}
$$

Insertion of (3.10), (3.11), and (3.12) into (3.9) yields

$$
\begin{aligned}
\int_{Q_{T} \times Q_{T}} & \left((u-v)^{+}\left(\partial_{t}+\partial_{s}\right) \phi+\sum_{i=1}^{d} \operatorname{sign}^{+}(u-v)\left[f_{i}(u)-f_{i}(v)\right.\right. \\
& -\left(\left|\partial_{x_{i}} A_{i}(u)\right|^{p_{i}-2} \partial_{x_{i}} A_{i}(u)\right. \\
& \left.\left.\left.-\left|\partial_{y_{i}} A_{i}(v)\right|^{p_{i}-2} \partial_{y_{i}} A_{i}(v)\right)\right]\left(\partial_{x_{i}}+\partial_{y_{i}}\right) \phi\right) \omega_{n} \omega_{l} d x d t d y d s \\
+ & \int_{Q_{T} \times(\{0\} \times \Omega)}\left(u_{0}-v\right)^{+} \phi_{n, l} d x d y d s \\
& +\int_{(\{0\} \times \Omega) \times Q_{T}}\left(u-v_{0}\right)^{+} \phi_{n, l} d x d t d y \geq \liminf _{\varepsilon \downarrow 0} E_{1}+E_{2},
\end{aligned}
$$

where

$$
\begin{aligned}
E_{1}= & \int_{Q_{T} \times Q_{T}} \sum_{i=1}^{d}\left(\operatorname{sign}_{\varepsilon}^{+}\right)^{\prime}(u-v)\left(\left|\partial_{x_{i}} \zeta(u)\right|^{p_{i}}+\left|\partial_{y_{i}} \zeta(v)\right|^{p_{i}}\right) \phi_{n, l} d x d t d y d s \\
E_{2}=- & \int_{Q_{T} \times Q_{T}} \sum_{i=1}^{d} \operatorname{sign}^{+}(u-v)\left|\partial_{x_{i}} A_{i}(u)\right|^{p_{i}-2} \partial_{x_{i}} A_{i}(u) \partial_{y_{i}} \phi_{n, l} d x d t d y d s \\
& \left.+\int_{Q_{T} \times Q_{T}} \sum_{i=1}^{d} \operatorname{sign}^{+}(u-v)\left|\partial_{y_{i}} A_{i}(v)\right|^{p_{i}-2} \partial_{y_{i}} A_{i}(v) \partial_{x_{i}} \phi_{n, l}\right) d x d t d y d s .
\end{aligned}
$$

Our goal now is to show that $\liminf _{\varepsilon \downarrow 0} E_{1}+E_{2} \geq 0$. Let us recall the following well known inequalities, which hold for any two real numbers $a, b$ and $p>1$ :

$$
\left(a|a|^{p-2}-b|b|^{p-2}\right)(a-b) \geq c(p)\left\{\begin{array}{ll}
|a-b|^{p}, & \text { if } p \geq 2 \\
\frac{|a-b|^{2}}{(|a|+|b|)^{2-p}}, & \text { if } 1<p<2
\end{array}\right\} \geq 0
$$

where $c(p)=2^{2-p}$ when $p \geq 2$ and $c(p)=p-1$ when $1<p<2$. Using first (3.14), then the chain rule (see Lemma 3.2 ), and finally integration by parts (there are no 
boundary terms since $\phi_{n, l}$ vanishes on the boundary), we get

$$
\begin{aligned}
& E_{1} \\
& \geq \int_{Q_{T} \times Q_{T}} \sum_{i=1}^{d}\left(\operatorname{sign}_{\varepsilon}^{+}\right)^{\prime}(u-v)\left(\left|\partial_{x_{i}} \zeta_{i}(u)\right|^{p_{i}-2} \partial_{x_{i}} \zeta_{i}(u) \partial_{y_{i}} \zeta_{i}(v)\right. \\
& \left.+\left|\partial_{y_{i}} \zeta_{i}(v)\right|^{p_{i}-2} \partial_{y_{i}} \zeta_{i}(v) \partial_{x_{i}} \zeta_{i}(u)\right) \phi_{n, l} d x d t d y d s \\
& =\int_{Q_{T} \times Q_{T}} \sum_{i=1}^{d} \partial_{y_{i}} \int_{u}^{v}\left(\operatorname{sign}_{\varepsilon}^{+}\right)^{\prime}(u-\xi)\left(A_{i}^{\prime}(\xi)\right)^{\frac{p_{i}-1}{p_{i}}} d \xi \\
& \times\left|\partial_{x_{i}} \zeta_{i}(u)\right|^{p_{i}-2} \partial_{x_{i}} \zeta_{i}(u) \phi_{n, l} d x d t d y d s \\
& +\int_{Q_{T} \times Q_{T}} \sum_{i=1}^{d} \partial_{x_{i}} \int_{v}^{u}\left(\operatorname{sign}_{\varepsilon}^{+}\right)^{\prime}(\xi-v)\left(A_{i}^{\prime}(\xi)\right)^{\frac{p_{i}-1}{p_{i}}} d \xi \\
& \times\left|\partial_{y_{i}} \zeta_{i}(v)\right|^{p_{i}-2} \partial_{y_{i}} \zeta_{i}(v) \phi_{n, l} d x d t d y d s \\
& =-\int_{Q_{T} \times Q_{T}} \sum_{i=1}^{d} \int_{u}^{v}\left(\operatorname{sign}_{\varepsilon}^{+}\right)^{\prime}(u-\xi)\left(A_{i}^{\prime}(\xi)\right)^{\frac{p_{i}-1}{p_{i}}} d \xi \\
& \times\left|\partial_{x_{i}} \zeta_{i}(u)\right|^{p_{i}-2} \partial_{x_{i}} \zeta_{i}(u) \partial_{y_{i}} \phi_{n, l} d x d t d y d s \\
& -\int_{Q_{T} \times Q_{T}} \sum_{i=1}^{d} \int_{v}^{u}\left(\operatorname{sign}_{\varepsilon}^{+}\right)^{\prime}(\xi-v)\left(A_{i}^{\prime}(\xi)\right)^{\frac{p_{i}-1}{p_{i}}} d \xi \\
& \times\left|\partial_{y_{i}} \zeta_{i}(v)\right|^{p_{i}-2} \partial_{y_{i}} \zeta_{i}(v) \partial_{x_{i}} \phi_{n, l} d x d t d y d s .
\end{aligned}
$$

To continue we need a technical lemma.

Lemma 3.4. Suppose $h \in L_{\mathrm{loc}}^{\infty}(\mathbf{R})$.

(i) Let $b \in \mathbf{R}$ be arbitrary but fixed. For a.e. $a \in \mathbf{R}$,

$$
\lim _{\varepsilon \downarrow 0} \int_{a}^{b}\left(\operatorname{sign}_{\varepsilon}^{+}\right)^{\prime}(a-\xi) h(\xi) d \xi=-\operatorname{sign}^{+}(a-b) h(a) .
$$

(ii) Let $a \in \mathbf{R}$ be arbitrary but fixed. For a.e. $b \in \mathbf{R}$,

$$
\lim _{\varepsilon \downarrow 0} \int_{b}^{a}\left(\operatorname{sign}_{\varepsilon}^{+}\right)^{\prime}(\xi-b) h(\xi) d \xi=\operatorname{sign}^{+}(a-b) h(b) .
$$

Proof. (i) Let $\xi=a$ be a Lebesgue point of $h$ and suppose $b<a$ (if $b>a$ there is nothing to prove). For any sufficiently small $\varepsilon$, it follows that

$$
\begin{aligned}
\int_{a}^{b}\left(\operatorname{sign}_{\varepsilon}^{+}\right)^{\prime}(a-\xi) h(\xi) d \xi= \\
\quad=-\int_{a-\varepsilon}^{a} \frac{\pi}{2 \varepsilon} \cos \left(\frac{\pi}{2 \varepsilon}(a-\xi)\right) h(\xi) d \xi=-\int_{0}^{\varepsilon} \frac{\pi}{2 \varepsilon} \cos \left(\frac{\pi}{2 \varepsilon} \xi\right) h(a-\xi) d \xi \\
\quad=-h(a)+\int_{0}^{\varepsilon} \frac{\pi}{2 \varepsilon} \cos \left(\frac{\pi}{2 \varepsilon} \xi\right)(h(a)-h(a-\xi)) d \xi
\end{aligned}
$$

where we have used that

$$
\int_{0}^{\varepsilon} \frac{\pi}{2 \varepsilon} \cos \left(\frac{\pi}{2 \varepsilon} \xi\right) d \xi=1
$$


This proves (i), since

$$
\begin{aligned}
& \left|\int_{0}^{\varepsilon} \frac{\pi}{2 \varepsilon} \cos \left(\frac{\pi}{2 \varepsilon} \xi\right)(h(a)-h(a-\xi)) d \xi\right| \\
& \quad \leq C \frac{1}{\varepsilon} \int_{0}^{\varepsilon}|h(a)-h(a-\xi)| d \xi \rightarrow 0 \quad \text { as } \varepsilon \downarrow 0 .
\end{aligned}
$$

(ii) Let $\xi=b$ be a Lebesgue point of $h$ and suppose $b<a$. As above, we find for any sufficiently small $\varepsilon$ that

$$
\begin{aligned}
& \int_{b}^{a}\left(\operatorname{sign}_{\varepsilon}^{+}\right)^{\prime}(\xi-b) h(\xi) d \xi \\
& \quad=h(b)+\int_{0}^{\varepsilon} \frac{\pi}{2 \varepsilon} \cos \left(\frac{\pi}{2 \varepsilon} \xi\right)(h(b+\xi)-h(b)) d \xi
\end{aligned}
$$

and (ii) follows by sending $\varepsilon \downarrow 0$.

As $p_{i}>1$ and thus $h(\xi):=\left(A_{i}^{\prime}(\xi)\right)^{\frac{p_{i}-1}{p_{i}}}$ is a locally bounded function, we can use Lemma 3.4 and (3.4) when taking the limit $\varepsilon \downarrow 0$ in (3.15). The result is

$$
\begin{aligned}
& \liminf _{\varepsilon \downarrow 0} E_{1} \\
& \geq \int_{Q_{T} \times Q_{T}} \sum_{i=1}^{d} \operatorname{sign}^{+}(u-v)\left(A_{i}^{\prime}(u)\right)^{\frac{p_{i}-1}{p_{i}}}\left|\partial_{x_{i}} \zeta_{i}(u)\right|^{p_{i}-2} \partial_{x_{i}} \zeta_{i}(u) \\
& \times \partial_{y_{i}} \phi_{n, l} d x d t d y d s \\
& \quad-\int_{Q_{T} \times Q_{T}} \sum_{i=1}^{d} \operatorname{sign}^{+}(u-v)\left(A_{i}^{\prime}(v)\right)^{\frac{p_{i}-1}{p_{i}}}\left|\partial_{y_{i}} \zeta_{i}(v)\right|^{p_{i}-2} \partial_{y_{i}} \zeta_{i}(v) \\
& \times \partial_{x_{i}} \phi_{n, l} d x d t d y d s .
\end{aligned}
$$

By the chain rule in Lemma 3.2,

$$
\begin{aligned}
& \left(A_{i}^{\prime}(u)\right)^{\frac{p_{i}-1}{p_{i}}}\left|\partial_{x_{i}} \zeta_{i}(u)\right|^{p_{i}-2} \partial_{x_{i}} \zeta_{i}(u) \\
& =\left|\left(A_{i}^{\prime}(u)\right)^{\frac{1}{p_{i}}} \partial_{x_{i}} \zeta_{i}(u)\right|^{p_{i}-2}\left(A_{i}^{\prime}(u)\right)^{\frac{1}{p_{i}}} \partial_{x_{i}} \zeta_{i}(u) \\
& =\left|\partial_{x_{i}} \zeta_{i}^{\left(A_{i}^{\prime}(\cdot)\right)^{\frac{1}{p_{i}}}}(u)\right|^{p_{i}-2} \partial_{x_{i}} \zeta_{i}^{\left(A_{i}^{\prime}(\cdot)\right)^{\frac{1}{p_{i}}}}(u) \\
& =\left|\partial_{x_{i}} A_{i}(u)\right|^{p_{i}-2} \partial_{x_{i}} A_{i}(u),
\end{aligned}
$$

which holds a.e. in $Q_{T}$ and in $L^{p_{i}^{\prime}}\left(Q_{T}\right) \cap L^{1}\left(Q_{T}\right), p_{i}^{\prime}=p_{i} /\left(p_{i}-1\right)$. Similarly,

$$
\left(A_{i}^{\prime}(v)\right)^{\frac{p_{i}-1}{p_{i}}}\left|\partial_{y_{i}} \zeta_{i}(v)\right|^{p_{i}-2} \partial_{y_{i}} \zeta_{i}(v)=\left|\partial_{y_{i}} A_{i}(v)\right|^{p_{i}-2} \partial_{y_{i}} A_{i}(v) .
$$

Hence, using this in (3.16), we finally obtain

$$
\begin{aligned}
& \underset{\varepsilon \downarrow 0}{\liminf _{\varepsilon \downarrow}} E_{1} \\
& \geq \int_{Q_{T} \times Q_{T}} \sum_{i=1}^{d} \operatorname{sign}^{+}(u-v)\left|\partial_{x_{i}} A_{i}(u)\right|^{p_{i}-2} \partial_{x_{i}} A_{i}(u) \partial_{y_{i}} \phi_{n, l} d x d t d y d s \\
& \quad-\int_{Q_{T} \times Q_{T}} \sum_{i=1}^{d} \operatorname{sign}^{+}(u-v)\left|\partial_{y_{i}} A_{i}(v)\right|^{p_{i}-2} \partial_{y_{i}} A_{i}(v) \partial_{x_{i}} \phi_{n, l} d x d t d y d s,
\end{aligned}
$$

and it follows that $\liminf \operatorname{ind}_{\varepsilon} E_{1}+E_{2} \geq 0$. 
Summarizing, from (3.13) we get

$$
\begin{aligned}
& \int_{Q_{T} \times Q_{T}}\left(I_{\text {time }}+I_{\text {conv }}+I_{\text {diff }}\right) \omega_{n} \omega_{l} d x d t d y d s \\
& +\int_{Q_{T} \times(\{0\} \times \Omega)}\left(u_{0}-v\right)^{+} \phi \omega_{n} \omega_{l} d x d y d s \\
& \quad+\int_{(\{0\} \times \Omega) \times Q_{T}}\left(u-v_{0}\right)^{+} \phi \omega_{n} \omega_{l} d x d t d y \geq 0,
\end{aligned}
$$

where

$$
\begin{aligned}
I_{\mathrm{time}} & =(u-v)^{+}\left(\partial_{t}+\partial_{s}\right) \phi \\
I_{\mathrm{conv}} & =\sum_{i=1}^{d} \operatorname{sign}^{+}(u-v)\left(f_{i}(u)-f_{i}(v)\right)\left(\partial_{x_{i}}+\partial_{y_{i}}\right) \phi \\
I_{\mathrm{diff}} & =-\sum_{i=1}^{d} \operatorname{sign}^{+}(u-v)\left(\left|\partial_{x_{i}} A_{i}(u)\right|^{p_{i}-2} \partial_{x_{i}} A_{i}(u)\right. \\
& \left.\quad-\left|\partial_{y_{i}} A_{i}(v)\right|^{p_{i}-2} \partial_{y_{i}} A_{i}(v)\right)\left(\partial_{x_{i}}+\partial_{y_{i}}\right) \phi .
\end{aligned}
$$

It takes a standard argument to send $n, l \uparrow \infty$ in the first integral in (3.17), see $[31,13]$. However, we have to be more careful with the remaining two integrals, since the entropy solutions are not assumed to be continuous in time with values in $L^{1}(\Omega)$. We shall proceed as in [13], but see also [46,35] and the references therein.

Let us denote the second and third integrals in (3.17) by $I_{u_{0}, v}(l, n)$ and $I_{u, v_{0}}(l, n)$, respectively. To handle $I_{u_{0}, v}(l, n)$, introduce the function

$$
\phi_{l}(s, x, y)=\int_{s}^{T} \phi\left(\frac{\tau}{2}, \frac{x+y}{2}\right) \omega_{l}\left(-\frac{\tau}{2}\right) d \tau .
$$

Since $\partial_{s} \phi_{l}=-\phi\left(\frac{s}{2}, \frac{x+y}{2}\right) \omega_{l}\left(-\frac{s}{2}\right)$, we may write

$$
I_{u_{0}, v}(l, n):=-\int_{Q_{T} \times(\{0\} \times \Omega)}\left(u_{0}-v\right)^{+} \partial_{s}\left(\phi_{l} \omega_{n}\right) d x d y d s,
$$

which enables us to use the entropy inequality for $v(s, y)$ to obtain an upper bound on $I_{u_{0}, v}(l, n)$ of the form

$$
\begin{aligned}
& I_{u_{0}, v}(l, n) \\
& \leq \int_{((0,2 / l) \times \Omega) \times(\{0\} \times \Omega)} H_{n} d x d y d s+\int_{(\{0\} \times \Omega) \times(\{0\} \times \Omega)}\left(u_{0}-v_{0}\right)^{+} \phi_{l} \omega_{n} d x d y,
\end{aligned}
$$

for some integrable function $H_{n}$ (independent of $l$ ). Here we have also used the fact that $\phi_{l} \equiv 0$ if $s>2 / l$

Sending $l \uparrow \infty$, the first term on the right-hand side tends to zero and regarding the second term observe that $\phi_{l}(0, x, y) \rightarrow \frac{1}{2} \phi\left(0, \frac{x+y}{2}\right)$. Hence

$$
\begin{aligned}
\limsup _{n \uparrow \infty} \limsup _{l \uparrow \infty} I_{u_{0}, v}(l, n) & \leq \frac{1}{2} \lim _{n \uparrow \infty} \int_{(\{0\} \times \Omega) \times(\{0\} \times \Omega)}\left(u_{0}-v_{0}\right)^{+} \phi \omega_{n} d x d y \\
& =\frac{1}{2} \int_{(\{0\} \times \Omega) \times(\{0\} \times \Omega)}\left(u_{0}-v_{0}\right)^{+} \phi d x,
\end{aligned}
$$

where (after the last equality sign) $u_{0}, v_{0}$ depend only on $x$.

Similarly, to handle $I_{u, v_{0}}$, we introduce the function

$$
\phi_{l}(t, x, y)=\int_{t}^{T} \phi\left(\frac{\tau}{2}, \frac{x+y}{2}\right) \omega_{l}\left(\frac{\tau}{2}\right) d \tau
$$


and proceed as before using the entropy inequality for $u(t, x)$. The result is

$$
\limsup _{n \uparrow \infty} \limsup _{l \uparrow \infty} I_{u, v_{0}}(l, n) \leq \frac{1}{2} \int_{(\{0\} \times \Omega) \times(\{0\} \times \Omega)}\left(u_{0}-v_{0}\right)^{+} \phi d x
$$

where $u_{0}, v_{0}, \phi$ depend only on $x$. This concludes the proof of Lemma 3.3.

We prove next that the conclusion of Lemma 3.3 continues to hold for test functions $\phi$ that do not vanish on the boundary.

Lemma 3.5. Let $u, v$ be entropy solutions of (1.1) with initial data $u_{0}, v_{0} \in L^{\infty}(\Omega)$, respectively. Then, for any $0 \leq \phi \in \mathcal{D}([0, T) \times \bar{\Omega})$, (3.6) holds.

Proof. We combine ideas from the proof of the previous lemma and Carrillo's method for handling Dirichlet boundary conditions [13].

Let $\phi=\phi(t, x, s, y)$ be any nonnegative function such that

$$
\begin{aligned}
& (t, x) \mapsto \phi(t, x, s, y) \in \mathcal{D}((0, T) \times \bar{\Omega}) \text { for any }(s, y) \in Q_{T} \\
& (s, y) \mapsto \phi(t, x, s, y) \in \mathcal{D}([0, T) \times \Omega) \text { for any }(t, x) \in Q_{T}
\end{aligned}
$$

We will start as in the proof of Lemma 3.3. However, since $\phi(\cdot, \cdot, s, y)$ does not vanish on the boundary, we have restrictions on the choice of $c$ in (2.6). Where we in the proof of Lemma 3.3 chose $c=v(s, y)$, we now choose $c=v^{+}(s, y)$. The result is (compare with $(3.7)$ )

$$
\begin{gathered}
\int_{Q_{T} \times Q_{T}}\left(\left(u-v^{+}\right)^{+} \partial_{t} \phi+\sum_{i=1}^{d} \operatorname{sign}^{+}\left(u-v^{+}\right)\left(f_{i}(u)-f_{i}\left(v^{+}\right)\right) \partial_{x_{i}} \phi\right. \\
\left.\quad-\sum_{i=1}^{d} \operatorname{sign}^{+}\left(u-v^{+}\right)\left|\partial_{x_{i}} A_{i}(u)\right|^{p_{i}-2} \partial_{x_{i}} A_{i}(u) \partial_{x_{i}} \phi\right) d x d t d y d s \\
\geq \liminf _{\varepsilon \downarrow 0} \int_{Q_{T}} \sum_{i=1}^{d}\left(\operatorname{sign}_{\varepsilon}^{+}\right)^{\prime}\left(u-v^{+}\right)\left|\partial_{x_{i}} \zeta_{i}(u)\right|^{p_{i}} \phi d x d t d y d s .
\end{gathered}
$$

$\operatorname{As~sign}^{+}\left(u-v^{+}\right)=0$ for $u \leq 0$, we deduce from (3.19) that

$$
\begin{gathered}
\int_{Q_{T} \times Q_{T}}\left(\left(u^{+}-v^{+}\right)^{+} \partial_{t} \phi+\sum_{i=1}^{d} \operatorname{sign}^{+}\left(u^{+}-v^{+}\right)\left(f_{i}\left(u^{+}\right)-f_{i}\left(v^{+}\right)\right) \partial_{x_{i}} \phi\right. \\
\left.\quad-\sum_{i=1}^{d} \operatorname{sign}^{+}\left(u^{+}-v^{+}\right)\left|\partial_{x_{i}} A_{i}\left(u^{+}\right)\right|^{p_{i}-2} \partial_{x_{i}} A_{i}\left(u^{+}\right) \partial_{x_{i}} \phi\right) d x d t d y d s \\
\geq \liminf _{\varepsilon \downarrow 0} \int_{Q_{T}} \sum_{i=1}^{d}\left(\operatorname{sign}_{\varepsilon}^{+}\right)^{\prime}\left(u^{+}-v^{+}\right)\left|\partial_{x_{i}} \zeta_{i}\left(u^{+}\right)\right|^{p_{i}} \phi d x d t d y d s .
\end{gathered}
$$


Next, where we in the proof of Lemma 3.3 chose $c=u(t, x)$, we now choose $c=u^{+}(t, x)$. The result is (compare with (3.8))

$$
\begin{aligned}
& \int_{Q_{T} \times Q_{T}}\left(\left(u^{+}-v\right)^{+} \partial_{s} \phi+\sum_{i=1}^{d} \operatorname{sign}^{+}\left(u^{+}-v\right)\left(f_{i}\left(u^{+}\right)-f_{i}(v)\right) \partial_{y_{i}} \phi\right. \\
& \left.\quad+\sum_{i=1}^{d} \operatorname{sign}^{+}\left(u^{+}-v\right)\left|\partial_{y_{i}} A_{i}(v)\right|^{p_{i}-2} \partial_{y_{i}} A_{i}(v) \partial_{y_{i}} \phi\right) d x d t d y d s \\
& +\int_{(\{0\} \times \Omega) \times Q_{T}}\left(u^{+}-v_{0}\right)^{+} \phi d x d t d y \\
& \geq \liminf _{\varepsilon \downarrow 0} \int_{Q_{T}} \sum_{i=1}^{d}\left(\operatorname{sign}_{\varepsilon}^{+}\right)^{\prime}\left(u^{+}-v\right)\left|\partial_{y_{i}} \zeta_{i}(v)\right|^{p_{i}} \phi d x d t d y d s .
\end{aligned}
$$

One checks easily that

$$
\operatorname{sign}^{+}\left(u^{+}-v\right)=\operatorname{sign}^{+}\left(u^{+}-v^{+}\right)\left(1-\operatorname{sign}^{+}\left(v^{-}\right)\right)+\operatorname{sign}^{+}\left(v^{-}\right) .
$$

Using this fact, we have

$$
\begin{aligned}
\operatorname{sign}^{+} & \left(u^{+}-v\right)\left(f_{i}\left(u^{+}\right)-f_{i}(v)\right) \\
= & \operatorname{sign}^{+}\left(u^{+}-v^{+}\right)\left(f_{i}\left(u^{+}\right)-f_{i}\left(v^{+}\right)\right) \mathbf{1}_{v \geq 0}+\operatorname{sign}^{+}\left(v^{-}\right)\left(f_{i}\left(u^{+}\right)-f_{i}(v)\right) \\
= & \operatorname{sign}^{+}\left(u^{+}-v^{+}\right)\left(f_{i}\left(u^{+}\right)-f_{i}\left(v^{+}\right)\right)-\operatorname{sign}^{+}\left(v^{-}\right) f_{i}(v) \\
& \quad+\operatorname{sign}^{+}\left(v^{-}\right) f_{i}\left(u^{+}\right)-\operatorname{sign}^{+}\left(u^{+}-v^{+}\right)\left(f_{i}\left(u^{+}\right)-f_{i}\left(v^{+}\right)\right) \mathbf{1}_{v<0} \\
= & \operatorname{sign}^{+}\left(u^{+}-v^{+}\right)\left(f_{i}\left(u^{+}\right)-f_{i}\left(v^{+}\right)\right)-\operatorname{sign}^{+}\left(v^{-}\right) f_{i}(v) .
\end{aligned}
$$

Similarly,

$$
\begin{aligned}
& \left(u^{+}-v\right)^{+}=\left(u^{+}-v^{+}\right)^{+}-\operatorname{sign}^{+}\left(v^{-}\right) v, \\
& \left(u^{+}-v_{0}\right)^{+}=\left(u^{+}-v_{0}^{+}\right)^{+}-\operatorname{sign}^{+}\left(v_{0}^{-}\right) v_{0},
\end{aligned}
$$

and

$$
\begin{aligned}
\operatorname{sign}^{+} & \left(u^{+}-v\right)\left|\partial_{y_{i}} A_{i}(v)\right|^{p_{i}-2} \partial_{y_{i}} A_{i}(v) \\
= & \operatorname{sign}^{+}\left(u^{+}-v^{+}\right)\left|\partial_{y_{i}} A_{i}\left(v^{+}\right)\right|^{p_{i}-2} \partial_{y_{i}} A_{i}\left(v^{+}\right) \\
& \quad+\operatorname{sign}^{+}\left(v^{-}\right)\left|\partial_{y_{i}} A_{i}(v)\right|^{p_{i}-2} \partial_{y_{i}} A_{i}(v) .
\end{aligned}
$$


Thus it follows from (3.21) and (3.22) that

$$
\begin{aligned}
& \int_{Q_{T} \times Q_{T}}\left(\left(u^{+}-v^{+}\right)^{+} \partial_{s} \phi+\sum_{i=1}^{d} \operatorname{sign}^{+}\left(u^{+}-v^{+}\right)\left(f_{i}\left(u^{+}\right)-f_{i}\left(v^{+}\right)\right) \partial_{y_{i}} \phi\right. \\
& \left.\quad+\sum_{i=1}^{d} \operatorname{sign}^{+}\left(u^{+}-v^{+}\right)\left|\partial_{y_{i}} A_{i}\left(v^{+}\right)\right|^{p_{i}-2} \partial_{y_{i}} A_{i}\left(v^{+}\right) \partial_{y_{i}} \phi\right) d x d t d y d s \\
& \quad+\int_{(\{0\} \times \Omega) \times Q_{T}}\left(u^{+}-v_{0}^{+}\right)^{+} \phi d x d t d y \\
& \geq \liminf _{\varepsilon \downarrow 0} \int_{Q_{T}} \sum_{i=1}^{d}\left(\operatorname{sign}_{\varepsilon}^{+}\right)^{\prime}\left(u^{+}-v^{+}\right)\left|\partial_{y_{i}} \zeta_{i}\left(v^{+}\right)\right|^{p_{i}} \phi d x d t d y d s \\
& \quad+\liminf _{\varepsilon \downarrow 0} \int_{Q_{T} \times Q_{T}} \sum_{i=1}^{d}\left(\operatorname{sign}^{+}\right)^{\prime}\left(u^{+}-v^{-}\right)\left|\partial_{y_{i}} \zeta_{i}\left(-v^{-}\right)\right|^{p_{i}} \phi d x d t d y d s \\
& \quad+\widetilde{\mathcal{L}}_{v}(\phi) \\
& \geq \liminf _{\varepsilon \downarrow 0} \int_{Q_{T} \times Q_{T}} \sum_{i=1}^{d}\left(\operatorname{sign}_{\varepsilon}^{+}\right)^{\prime}\left(u^{+}-v^{+}\right)\left|\partial_{y_{i}} \zeta_{i}\left(v^{+}\right)\right|^{p_{i}} \phi d x d t d y d s \\
& \quad+\widetilde{\mathcal{L}}_{v}(\phi),
\end{aligned}
$$

where

$$
\begin{aligned}
\widetilde{\mathcal{L}}_{v}(\phi):= & \int_{Q_{T} \times Q_{T}} \operatorname{sign}^{+}\left(v^{-}\right)\left(v \partial_{s} \phi+\right. \\
& -\sum_{i=1}^{d} f_{i}(v) \partial_{y_{i}} \phi \\
& \left.+\sum_{i=1}^{d}\left|\partial_{y_{i}} A_{i}(v)\right|^{p_{i}-2} \partial_{y_{i}} A_{i}(v) \partial_{y_{i}} \phi\right) d x d t d y d s \\
& \int_{(\{0\} \times \Omega) \times Q_{T}} \operatorname{sign}^{+}\left(v_{0}^{-}\right) v_{0} \phi d x d t d y .
\end{aligned}
$$

Adding (3.20) and (3.23) yields (compare with (3.13))

$$
\begin{gathered}
\int_{Q_{T} \times Q_{T}}\left(\left(u^{+}-v^{+}\right)^{+}\left(\partial_{t}+\partial_{s}\right) \phi+\sum_{i=1}^{d} \operatorname{sign}^{+}\left(u^{+}-v^{+}\right)\left[f_{i}\left(u^{+}\right)-f_{i}\left(v^{+}\right)\right.\right. \\
-\left(\left|\partial_{x_{i}} A_{i}\left(u^{+}\right)\right|^{p_{i}-2} \partial_{x_{i}} A_{i}\left(u^{+}\right)\right. \\
\left.\left.\left.-\left|\partial_{y_{i}} A_{i}\left(v^{+}\right)\right|^{p_{i}-2} \partial_{y_{i}} A_{i}\left(v^{+}\right)\right)\right]\left(\partial_{x_{i}}+\partial_{y_{i}}\right) \phi\right) d x d t d y d s \\
+\int_{(\{0\} \times \Omega) \times Q_{T}}\left(u^{+}-v_{0}^{+}\right)^{+} \phi d x d t d y \geq \liminf _{\varepsilon \downarrow 0} E_{1}+E_{2}+\widetilde{\mathcal{L}}_{v}(\phi),
\end{gathered}
$$


where

$$
\begin{aligned}
E_{1}= & \int_{Q_{T} \times Q_{T}} \sum_{i=1}^{d}\left(\operatorname{sign}_{\varepsilon}^{+}\right)^{\prime}\left(u^{+}-v^{+}\right) \\
& \times\left(\left|\partial_{x_{i}} \zeta\left(u^{+}\right)\right|^{p_{i}}+\left|\partial_{y_{i}} \zeta\left(v^{+}\right)\right|^{p_{i}}\right) \phi d x d t d y d s \\
E_{2}= & -\int_{Q_{T} \times Q_{T}} \sum_{i=1}^{d} \operatorname{sign}^{+}\left(u^{+}-v^{+}\right)\left|\partial_{x_{i}} A_{i}\left(u^{+}\right)\right|^{p_{i}-2} \partial_{x_{i}} A_{i}\left(u^{+}\right) \partial_{y_{i}} \phi d x d t d y d s \\
& \left.+\int_{Q_{T} \times Q_{T}} \sum_{i=1}^{d} \operatorname{sign}^{+}\left(u^{+}-v^{+}\right)\left|\partial_{y_{i}} A_{i}\left(v^{+}\right)\right|^{p_{i}-2} \partial_{y_{i}} A_{i}\left(v^{+}\right) \partial_{x_{i}} \phi\right) d x d t d y d s .
\end{aligned}
$$

As in the proof of Lemma 3.3, our goal now is to show that $\lim \inf _{\varepsilon \downarrow 0} E_{1}+E_{2} \geq 0$. However, different from the proof of Lemma 3.3, the test function $\phi(\cdot, \cdot, s, y)$ does not vanish on the boundary $(0, T) \times \partial \Omega$, so it becomes necessary to invoke the integration by parts rule (D.4), which is stated for $u$ not $u^{+}$. But (D.4) also applies to $u^{+}$. Indeed, this is true because $\zeta\left(u^{+}\right)=\zeta^{\psi}(u), \psi(\xi):=\operatorname{sign}^{+}(\xi) \in L^{\infty}(\mathbf{R})$, for $i=1, \ldots, d$. Using first (3.14), then the chain rule (3.5), and finally integration by parts (D.4), we can calculate as follows:

$$
\begin{aligned}
& E_{1} \\
& \geq \int_{Q_{T} \times Q_{T}} \sum_{i=1}^{d}\left(\operatorname{sign}_{\varepsilon}^{+}\right)^{\prime}\left(u^{+}-v^{+}\right)\left(\left|\partial_{x_{i}} \zeta_{i}\left(u^{+}\right)\right|^{p_{i}-2} \partial_{x_{i}} \zeta_{i}\left(u^{+}\right) \partial_{y_{i}} \zeta_{i}\left(v^{+}\right)\right. \\
& \left.+\left|\partial_{y_{i}} \zeta_{i}\left(v^{+}\right)\right|^{p_{i}-2} \partial_{y_{i}} \zeta_{i}\left(v^{+}\right) \partial_{x_{i}} \zeta_{i}\left(u^{+}\right)\right) \phi d x d t d y d s \\
& =\int_{Q_{T} \times Q_{T}} \sum_{i=1}^{d} \partial_{y_{i}} \int_{u^{+}}^{v^{+}}\left(\operatorname{sign}_{\varepsilon}^{+}\right)^{\prime}\left(u^{+}-\xi\right)\left(A_{i}^{\prime}(\xi)\right)^{\frac{p_{i}-1}{p_{i}}} d \xi \\
& \times\left|\partial_{x_{i}} \zeta_{i}\left(u^{+}\right)\right|^{p_{i}-2} \partial_{x_{i}} \zeta_{i}\left(u^{+}\right) \phi d x d t d y d s \\
& +\int_{Q_{T} \times Q_{T}} \sum_{i=1}^{d} \partial_{x_{i}} \int_{v^{+}}^{u^{+}}\left(\operatorname{sign}_{\varepsilon}^{+}\right)^{\prime}\left(\xi-v^{+}\right)\left(A_{i}^{\prime}(\xi)\right)^{\frac{p_{i}-1}{p_{i}}} d \xi \\
& \times\left|\partial_{y_{i}} \zeta_{i}\left(v^{+}\right)\right|^{p_{i}-2} \partial_{y_{i}} \zeta_{i}\left(v^{+}\right) \phi d x d t d y d s \\
& =\int_{Q_{T} \times Q_{T}} \sum_{i=1}^{d} \int_{u^{+}}^{v^{+}}\left(\operatorname{sign}_{\varepsilon}^{+}\right)^{\prime}\left(u^{+}-\xi\right)\left(A_{i}^{\prime}(\xi)\right)^{\frac{p_{i}-1}{p_{i}}} d \xi \\
& \times\left|\partial_{x_{i}} \zeta_{i}\left(u^{+}\right)\right|^{p_{i}-2} \partial_{x_{i}} \zeta_{i}\left(u^{+}\right) \partial_{y_{i}} \phi d x d t d y d s \\
& \sum_{\sum_{i=1}^{d}}^{u_{Q^{+}}}\left(\operatorname{sign}_{v^{+}}^{+}\right)^{\prime}\left(\xi-v^{+}\right)\left(A_{i}^{\prime}(\xi)\right)^{\frac{p_{i}-1}{p_{i}}} d \xi \\
& \times\left|\partial_{y_{i}} \zeta_{i}\left(v^{+}\right)\right|^{p_{i}-2} \partial_{y_{i}} \zeta_{i}\left(v^{+}\right) \partial_{x_{i}} \phi d x d t d y d s .
\end{aligned}
$$

We now proceed exactly as in the proof of Lemma 3.3 by applying Lemma 3.4 when passing to the limit $\varepsilon \downarrow 0$ in (3.25) and then using the chain rule (3.5). The result 
is

$$
\begin{aligned}
& \liminf _{\varepsilon \downarrow 0} E_{1} \\
& \geq \int_{Q_{T} \times Q_{T}} \sum_{i=1}^{d} \operatorname{sign}^{+}\left(u^{+}-v^{+}\right)\left|\partial_{x_{i}} A_{i}\left(u^{+}\right)\right|^{p_{i}-2} \partial_{x_{i}} A_{i}\left(u^{+}\right) \partial_{y_{i}} \phi d x d t d y d s \\
& \quad-\int_{Q_{T} \times Q_{T}} \sum_{i=1}^{d} \operatorname{sign}^{+}\left(u^{+}-v^{+}\right)\left|\partial_{y_{i}} A_{i}\left(v^{+}\right)\right|^{p_{i}-2} \partial_{y_{i}} A_{i}\left(v^{+}\right) \partial_{x_{i}} \phi d x d t d y d s,
\end{aligned}
$$

from which it follows that $\liminf _{\varepsilon \downarrow 0} E_{1}+E_{2} \geq 0$.

Summarizing, we have established

$$
\begin{gathered}
\int_{Q_{T} \times Q_{T}}\left(\left(u^{+}-v^{+}\right)^{+}\left(\partial_{t}+\partial_{s}\right) \phi+\sum_{i=1}^{d} \operatorname{sign}^{+}\left(u^{+}-v^{+}\right)\left[f_{i}\left(u^{+}\right)-f_{i}\left(v^{+}\right)\right.\right. \\
-\left(\left|\partial_{x_{i}} A_{i}\left(u^{+}\right)\right|^{p_{i}-2} \partial_{x_{i}} A_{i}\left(u^{+}\right)\right. \\
\left.\left.\left.-\left|\partial_{y_{i}} A_{i}\left(v^{+}\right)\right|^{p_{i}-2} \partial_{y_{i}} A_{i}\left(v^{+}\right)\right)\right]\left(\partial_{x_{i}}+\partial_{y_{i}}\right) \phi\right) d x d t d y d s \\
+\int_{(\{0\} \times \Omega) \times Q_{T}}\left(u^{+}-v_{0}^{+}\right)^{+} \phi d x d t d y \geq \widetilde{\mathcal{L}}_{v}(\phi) .
\end{gathered}
$$

The remaining part of the proof is very similar to Carrillo [13], but we include it for the convenience of the reader. Let $B$ be a ball such that

$$
B \subset B^{\prime} \text { or } B \cap \partial \Omega=\emptyset,
$$

where $B^{\prime}$ is a ball such that

$B^{\prime} \cap \partial \Omega \subset\{(x, L(x)): x \in \Omega\}$ for some Lipschitz continuous function $L$.

Let us introduce a nonnegative function $\phi \in \mathcal{D}\left([0, T) \times \mathbf{R}^{d}\right)$ such that

$$
\operatorname{supp}(\phi) \cap[0, T) \times \mathbf{R}^{d} \subset[0, T) \times B .
$$

For $l \geq 1$, let $\omega_{l}: \mathbf{R} \rightarrow \mathbf{R}$ be a sequence of mollifiers such that $\operatorname{supp}\left(\omega_{l}\right)$ in $(-2 / l, 0)$. For $n \geq 1$, we can find a sequence of mollifiers $\omega_{n}: \mathbf{R}^{d} \rightarrow \mathbf{R}$ such that for $n$ large enough,

$$
y \mapsto \omega_{n}(y-x) \text { belongs to } \mathcal{D}(\Omega), \quad \forall x \in B .
$$

Define $\Theta_{n}(y)=\int_{\Omega} \omega_{n}(y-x) d x$. Then

$$
\begin{aligned}
& \text { for any } y, y^{\prime} \in B \text { such that } y \leq y^{\prime}, \Theta_{n}(y) \leq \Theta_{n}\left(y^{\prime}\right) \text {, and } \\
& \Theta_{n}(y)=1 \text { for any } y \in B \text { such that } d\left(y, \mathbf{R}^{d} \backslash \Omega\right)>\frac{c}{n},
\end{aligned}
$$

for some positive constant $c$ depending on $B$.

Define

$$
\phi_{n, l}(t, x, s, y)=\phi(s, y) \omega_{n}(y-x) \omega_{l}(s-t) .
$$

Note that for $n$ and $l$ large enough, $\phi_{n, l}$ satisfies (3.18). Moreover, the function

$$
\begin{aligned}
\phi_{n}(s, y) & :=\int_{Q_{T}} \phi_{n, l}(t, x, s, y) d x d t \\
& =\phi(s, y) \int_{\Omega} \omega_{n}(y-x) d x \int_{0}^{T} \omega_{l}(s-t) d t=\phi(s, y) \Theta_{n}(y)
\end{aligned}
$$


has the following properties:

$\phi_{n} \in \mathcal{D}([0, T) \times \Omega)$ for each $n, \phi_{n}$ is an increasing sequence, and for all $y$ such that $d\left(y, \mathbf{R}^{d} \backslash \Omega\right)>\frac{c}{n}, \phi_{n}(s, y)=\phi(s, y)$.

Note that

$$
\phi_{n} \leq \phi \text { and } \phi_{n} \uparrow \phi \text { in } L^{q}((0, T) \times \Omega) \text { as } n \uparrow \infty \text { for any } q \geq 1 \text {. }
$$

Substituting $\phi=\phi_{n, l}$ in (3.26) and using the identities

$$
\left(\partial_{t}+\partial_{s}\right) \phi_{n, l}=\partial_{s} \phi \omega_{n} \omega_{l}, \quad\left(\nabla_{x}+\nabla_{y}\right) \phi_{n, l}=\nabla_{y} \phi \omega_{n} \omega_{l},
$$

we deduce

$$
\begin{gathered}
\int_{Q_{T} \times Q_{T}}\left(\left(u^{+}-v^{+}\right)^{+} \partial_{s} \phi+\sum_{i=1}^{d} \operatorname{sign}^{+}\left(u^{+}-v^{+}\right)\left[f_{i}\left(u^{+}\right)-f_{i}\left(v^{+}\right)\right.\right. \\
-\left(\left|\partial_{x_{i}} A_{i}\left(u^{+}\right)\right|^{p_{i}-2} \partial_{x_{i}} A_{i}\left(u^{+}\right)\right. \\
\left.\left.\left.-\left|\partial_{y_{i}} A_{i}\left(v^{+}\right)\right|^{p_{i}-2} \partial_{y_{i}} A_{i}\left(v^{+}\right)\right) \partial_{y_{i}} \phi\right] \omega_{n} \omega_{l}\right) d x d t d y d s \\
+\int_{(\{0\} \times \Omega) \times Q_{T}}\left(u^{+}-v_{0}^{+}\right)^{+} \phi \omega_{n} \omega_{l} d x d t d y \geq \mathcal{L}_{v}\left(\phi_{n}\right),
\end{gathered}
$$

where

$$
\begin{aligned}
\mathcal{L}_{v}\left(\phi_{n}\right):= & \int_{Q_{T}} \operatorname{sign}^{+}\left(v^{-}\right)\left(v \partial_{s} \phi_{n}+\sum_{i=1}^{d} f_{i}(v) \partial_{y_{i}} \phi_{n}\right. \\
& \left.\quad-\sum_{i=1}^{d}\left|\partial_{y_{i}} A_{i}(v)\right|^{p_{i}-2} \partial_{y_{i}} A_{i}(v) \partial_{y_{i}} \phi_{n}\right) d y d s \\
& +\int_{\{0\} \times \Omega} \operatorname{sign}^{+}\left(v_{0}^{-}\right) v_{0} \phi_{n} d y \\
= & \int_{Q_{T}}\left((-v)^{+} \partial_{s} \phi_{n}-\sum_{i=1}^{d} \operatorname{sign}^{+}(-v) f_{i}(v) \partial_{y_{i}} \phi_{n}\right. \\
& +\sum_{\{0\} \times \Omega}\left(-v_{0}\right)^{+} \phi_{n} d y .
\end{aligned}
$$

Next we pass to the limit as $l, n \uparrow \infty$ in each term in (3.28). Sending $l, n \uparrow \infty$ in the first integral in (3.28) yields

$$
\begin{aligned}
\int_{Q_{T}} k_{+}(s, y)\left(\left(u^{+}-v^{+}\right) \partial_{s} \phi+\sum_{i=1}^{d}\left[f_{i}\left(u^{+}\right)-f_{i}\left(v^{+}\right)\right.\right. \\
\left.\left.\quad-\left(\left|\partial_{y_{i}} A_{i}\left(u^{+}\right)\right|^{p_{i}-2} \partial_{y_{i}} A_{i}\left(u^{+}\right)-\left|\partial_{y_{i}} A_{i}\left(v^{+}\right)\right|^{p_{i}-2} \partial_{y_{i}} A_{i}\left(v^{+}\right)\right)\right] \partial_{y_{i}} \phi\right) d y d s
\end{aligned}
$$


where $k_{+}(s, y) \in \widetilde{\operatorname{sign}}^{+}\left(u^{+}(s, y)-v^{+}(s, y)\right)$ and $\widetilde{\operatorname{sign}}^{+}(\xi)= \begin{cases}0, & \xi<0, \\ {[0,1],} & \xi=0, \\ 1, & \xi>0 .\end{cases}$

Introduce the function

$$
\varphi_{n, l}(t, x, s, y)=\phi(s, y) \omega_{n}(y-x) \int_{t}^{T} \omega_{l}(s-\tau) d \tau
$$

Then $\partial_{t} \varphi_{n, l}=-\phi(s, y) \omega_{n}(y-x) \omega_{l}(s-t)=-\phi \omega_{n} \omega_{l}$, and thus

$$
\begin{aligned}
& \int_{(\{0\} \times \Omega) \times Q_{T}}\left(u^{+}-v_{0}^{+}\right)^{+} \phi \omega_{n} \omega_{l} d x d t d y \\
& =-\int_{(\{0\} \times \Omega) \times Q_{T}}\left(u^{+}-v_{0}^{+}\right)^{+} \partial_{t} \varphi_{n, l} d x d t d y .
\end{aligned}
$$

We then exploit that $u=u(t, x)$ is an entropy solution to obtain

$$
\begin{aligned}
& \int_{(\{0\} \times \Omega) \times Q_{T}}\left(u^{+}-v_{0}^{+}\right)^{+} \phi \omega_{n} \omega_{l} d x d t d y \\
& =-\int_{(\{0\} \times \Omega) \times Q_{T}}\left(u^{+}-v_{0}^{+}\right)^{+} \partial_{t} \varphi_{n, l} d x d t d y \\
& \leq \int_{(\{0\} \times \Omega) \times Q_{T}} \sum_{i=1}^{d} \operatorname{sign}^{+}\left(u-v_{0}^{+}\right) \\
& \quad \times\left[f_{i}(u)-f_{i}\left(v^{+}\right)-\left|\partial_{x_{i}} A_{i}(u)\right|^{p_{i}-2} \partial_{x_{i}} A_{i}(u)\right] \partial_{x_{i}} \varphi_{n, l} d x d t d y \\
& \quad+\int_{(\{0\} \times \Omega)) \times(\{0\} \times \Omega)}\left(u_{0}-v_{0}^{+}\right)^{+} \phi \omega_{n} d x d y \\
& =\int_{(\{0\} \times \Omega) \times((0,2 / l) \times \Omega)} \sum_{i=1}^{d} \operatorname{sign}^{+}\left(u-v_{0}^{+}\right) \\
& \quad \times\left[f_{i}(u)-f_{i}\left(v^{+}\right)-\left|\partial_{x_{i}} A_{i}(u)\right|^{p_{i}-2} \partial_{x_{i}} A_{i}(u)\right] \phi \partial_{x_{i}} \omega_{n} \int_{t}^{T} \omega_{l}(-\tau) d \tau d x d y \\
& \quad+\int_{(\{0\} \times \Omega) \times(\{0\} \times \Omega)}\left(u_{0}-v_{0}^{+}\right)^{+} \omega_{n} \phi d x d y,
\end{aligned}
$$

where we have also used that $\int_{0}^{T} \omega_{l}(-\tau) d \tau=1$.

Clearly, the first integral on the right-hand side of the last equality in (3.30) vanishes as $l \uparrow \infty$. Hence it follows that

$$
\begin{aligned}
& \underset{n \uparrow \infty}{\limsup } \limsup _{l \uparrow \infty} \int_{(\{0\} \times \Omega) \times Q_{T}}\left(u^{+}-v_{0}^{+}\right)^{+} \phi \omega_{n} \omega_{l} d x d t d y \\
& \quad \leq \lim _{n \uparrow \infty} \int_{(\{0\} \times \Omega) \times(\{0\} \times \Omega)}\left(u_{0}-v_{0}^{+}\right)^{+} \omega_{n} \phi d x d y \\
& \quad=\int_{\{0\} \times \Omega}\left(u_{0}-v_{0}^{+}\right)^{+} \phi d y=\int_{\{0\} \times \Omega}\left(u_{0}^{+}-v_{0}^{+}\right)^{+} \phi d y .
\end{aligned}
$$

Since $v$ is an entropy solution and $\phi_{n} \uparrow \phi$ as $n \uparrow \infty$, the sequence $\mathcal{L}_{v}\left(\phi_{n}\right)$ is monotonically increasing as $n \uparrow \infty$ and $0 \leq \mathcal{L}_{v}\left(\phi_{n}\right) \leq \mathcal{L}_{v}(\phi)$, so it converges. From 
this, (3.29), and (3.30),

$$
\begin{gathered}
\int_{Q_{T}}\left(\left(u^{+}-v^{+}\right)^{+} \partial_{s} \phi+\sum_{i=1}^{d} \operatorname{sign}^{+}\left(u^{+}-v^{+}\right)\left[f_{i}\left(u^{+}\right)-f_{i}\left(v^{+}\right)\right.\right. \\
\left.\left.-\left(\left|\partial_{y_{i}} A_{i}\left(u^{+}\right)\right|^{p_{i}-2} \partial_{y_{i}} A_{i}\left(u^{+}\right)-\left|\partial_{y_{i}} A_{i}\left(v^{+}\right)\right|^{p_{i}-2} \partial_{y_{i}} A_{i}\left(v^{+}\right)\right)\right] \partial_{y_{i}} \phi\right) d y d s \\
\quad+\int_{(\{0\} \times \Omega)}\left(u_{0}^{+}-v_{0}^{+}\right)^{+} \phi d y \geq \lim _{n \uparrow \infty} \mathcal{L}_{v}\left(\phi_{n}\right),
\end{gathered}
$$

for any $0 \leq \phi=\phi(s, y) \in \mathcal{D}([0, T) \times B)$.

Observe that if $w$ is an entropy solution of (1.1) with initial data $w_{0}$, then $W:=-w$ is an entropy solution of

$$
\partial_{t} W-\sum_{i=1}^{d} \partial_{x_{i}} f_{i}(-W)=-\sum_{i=1}^{d} \partial_{x_{i}}\left(\left|\partial_{x_{i}} A_{i}(-W)\right|^{p_{i}-2} \partial_{x_{i}} A_{i}(-W)\right),
$$

with $\left.W\right|_{t=0}=-w_{0}$ and $\left.W\right|_{(0, T) \times \partial \Omega}=0$. Therefore, replacing $u, v, u_{0}$, and $v_{0}$ by $-v,-u,-v_{0}$, and $-u_{0}$, respectively, in the above calculations, we deduce the following version of (3.31):

$$
\begin{aligned}
& \int_{Q_{T} \times Q_{T}}\left(\left(v^{-}-u^{-}\right)^{+} \partial_{s} \phi-\sum_{i=1}^{d} \operatorname{sign}^{+}\left(v^{-}-u^{-}\right)\left[f_{i}\left(-v^{-}\right)-f_{i}\left(-u^{-}\right)\right.\right. \\
& \left.\left.-\left(\left|\partial_{y_{i}} A_{i}\left(-v^{-}\right)\right|^{p_{i}-2} \partial_{y_{i}} A_{i}\left(-v^{-}\right)-\left|\partial_{y_{i}} A_{i}\left(-u^{-}\right)\right|^{p_{i}-2} \partial_{y_{i}} A_{i}\left(-u^{-}\right)-\right)\right] \partial_{y_{i}} \phi\right) d y d s \\
& \quad+\int_{(\{0\} \times \Omega)}\left(v_{0}^{-}-u_{0}^{-}\right)^{+} \phi d y \geq-\lim _{n \uparrow \infty} \mathcal{L}_{u}\left(\phi_{n}\right),
\end{aligned}
$$

where

$$
\begin{gathered}
\mathcal{L}_{u}\left(\phi_{n}\right)=\int_{Q_{T}} \operatorname{sign}^{+}\left(u^{+}\right)\left(u \partial_{s} \phi_{n}+f_{i}(u) \partial_{y_{i}} \phi_{n}\right. \\
\left.\quad-\sum_{i=1}^{d}\left|\partial_{y_{i}} A_{i}(u)\right|^{p_{i}-2} \partial_{y_{i}} A_{i}(u) \partial_{y_{i}} \phi_{n}\right) d y d s \\
+\int_{(\{0\} \times \Omega)} \operatorname{sign}^{+}\left(u_{0}^{+}\right) u_{0} \phi_{n} d y .
\end{gathered}
$$

Adding (3.31) and (3.32) gives

$$
\begin{aligned}
\mathcal{E}(\phi):= & \int_{Q_{T} \times Q_{T}}\left((u-v)^{+} \partial_{s} \phi+\sum_{i=1}^{d} \operatorname{sign}^{+}(u-v)\left[f_{i}(u)-f_{i}(v)\right.\right. \\
& \left.\left.-\left(\left|\partial_{y_{i}} A_{i}(u)\right|^{p_{i}-2} \partial_{y_{i}} A_{i}(u)-\left|\partial_{y_{i}} A_{i}(v)\right|^{p_{i}-2} \partial_{y_{i}} A_{i}(v)\right)\right] \partial_{y_{i}} \phi\right) d y d s \\
& +\int_{(\{0\} \times \Omega)}\left(u_{0}-v_{0}\right)^{+} \phi d x \geq \lim _{n \uparrow \infty} \mathcal{L}_{v}\left(\phi_{n}\right)-\lim _{n \uparrow \infty} \mathcal{L}_{u}\left(\phi_{n}\right),
\end{aligned}
$$

for any nonnegative function $\phi=\phi(s, y) \in \mathcal{D}([0, T) \times B)$. 
Let $0 \leq \phi \in \mathcal{D}([0, T) \times B)$ be arbitrary. For $m \geq 1$, define the function $\phi_{m}(s, y):=\phi(s, y) \Theta_{m}(y), \Theta_{m}(y)=\int_{\Omega} \omega_{m}(y-x) d x$. As $\phi_{m} \in \mathcal{D}([0, T) \times B)$, Lemma 3.3 gives $\mathcal{E}\left(\phi_{m}\right) \geq 0$ for each $m$. Hence

$$
\begin{aligned}
\mathcal{E}(\phi) & =\mathcal{E}\left(\phi^{m}\right)+\mathcal{E}\left(\phi\left(1-\Theta_{m}\right)\right) \geq \mathcal{E}\left(\phi\left(1-\Theta_{m}\right)\right) \\
& \geq \lim _{n \uparrow \infty} \mathcal{L}_{v}\left(\phi \Theta_{n}\left(1-\Theta_{m}\right)\right)-\lim _{n \uparrow \infty} \mathcal{L}_{u}\left(\phi \Theta_{n}\left(1-\Theta_{m}\right)\right) .
\end{aligned}
$$

The right-hand side tends to zero when we send first $n \uparrow \infty$ and second $m \uparrow \infty$, which is a consequence of the fact that for each fixed $m$

$$
\Theta_{n}\left(1-\Theta_{m}\right)=\Theta_{n}-\Theta_{m}, \quad n>m .
$$

Hence we have proved that

$$
\mathcal{E}(\phi) \geq 0, \quad \forall \phi \in \mathcal{D}([0, T) \times B), \phi \geq 0 .
$$

Let $\Omega_{0} \subset \Omega$ be such that $\Omega_{0} \cup\left(\cup_{i=0}^{I} B_{i}\right)$ is a covering of $\Omega$, where $\left(B_{i}\right)_{1 \leq i \leq I}$ are balls satisfying (3.27). Let $(\varphi)_{0 \leq i \leq I}$ be a partition of unity related to the above covering, so that $\varphi_{0} \in \mathcal{D}(\Omega), \varphi_{i} \in \mathcal{D}\left(B_{i}\right), 1 \leq i \leq I$. Let $\phi \in \mathcal{D}([0, T) \times \bar{\Omega}), \phi \geq 0$, and $\phi_{i}:=\phi \varphi_{i}(1 \leq i \leq I)$. From (3.34),

$$
\mathcal{E}\left(\phi_{i}\right) \geq 0, \quad i=0, \ldots, I .
$$

By linearity of $\phi \mapsto \mathcal{L}(\phi)$, Lemma 3.5 follows by summing (3.35) over $i$.

Concluding the proof of Theorem 3.1. In Lemma 3.5, we pick a test function $\phi$ of the form

The result is

$$
\phi=\psi(t), \quad 0 \leq \psi \in \mathcal{D}([0, T))
$$

$$
-\int_{0}^{T} \psi^{\prime}(t)\left(\int_{\Omega}(u(t, x)-v(t, x))^{+} d x\right) d t \leq \int_{\Omega}\left(u_{0}-v_{0}\right)^{+} d x,
$$

from which Theorem 3.1 follows in a standard way.

\section{REFERENCES}

[1] H. W. Alt and S. Luckhaus. Quasilinear elliptic-parabolic differential equations. Math. Z., 183(3):311-341, 1983.

[2] D. Aregba-Driollet, R. Natalini, and S. Tang. Explicit diffusive kinetic schemes for nonlinear degenerate parabolic systems. Math. Comp., 73(245):63-94 (electronic), 2004.

[3] C. Bardos, A. Y. LeRoux, and J.-C. Nédélec. First order quasilinear equations with boundary conditions. Comm. Partial Differential Equations, 4(9):1017-1034, 1979.

[4] M. Bendahmane and K. H. Karlsen. Renormalized entropy solutions for quasi-linear anisotropic degenerate parabolic equations. SIAM J. Math. Anal. To appear.

[5] M. Bendahmane and K. H. Karlsen. Renormalized entropy solutions for doubly nonlinear anisotropic degenerate parabolic equations. Work in preparation, 2004.

[6] P. Bénilan and H. Touré. Sur l'équation générale $u_{t}=a\left(\cdot, u, \phi(\cdot, u)_{x}\right)_{x}+v$ dans $L^{1}$. II. Le problème d'évolution. Ann. Inst. H. Poincaré Anal. Non Linéaire, 12(6):727-761, 1995.

[7] P. Benilan and P. Wittbold. On mild and weak solutions of elliptic-parabolic problems. Adv. Differential Equations, 1(6):1053-1073, 1996.

[8] F. Bouchut. Renormalized solutions to the vlasov equation with coefficients of bounded variation. Arch. Rational Mech. Anal., 157:75-90, 2001.

[9] F. Bouchut, F. R. Guarguaglini, and R. Natalini. Diffusive BGK approximations for nonlinear multidimensional parabolic equations. Indiana Univ. Math. J., 49(2):723-749, 2000.

[10] R. Bürger, S. Evje, and K. H. Karlsen. On strongly degenerate convection-diffusion problems modeling sedimentation-consolidation processes. J. Math. Anal. Appl., 247(2):517-556, 2000.

[11] M. C. Bustos, F. Concha, R. Bürger, and E. M. Tory. Sedimentation and Thickening: Phenomenological Foundation and Mathematical Theory. Kluwer Academic Publishers, Dordrecht, The Netherlands, 1999.

[12] J. Carrillo. On the uniqueness of the solution of the evolution dam problem. Nonlinear Anal., 22(5):573-607, 1994.

[13] J. Carrillo. Entropy solutions for nonlinear degenerate problems. Arch. Rational Mech. Anal., 147(4):269-361, 1999. 
[14] J. Carrillo and P. Wittbold. Uniqueness of renormalized solutions of degenerate ellipticparabolic problems. J. Differential Equations, 156(1):93-121, 1999.

[15] G.-Q. Chen and E. DiBenedetto. Stability of entropy solutions to the Cauchy problem for a class of nonlinear hyperbolic-parabolic equations. SIAM J. Math. Anal., 33(4):751-762 (electronic), 2001.

[16] G.-Q. Chen and K. H. Karlsen. $L^{1}$ framework for continuous dependence and error estimates for quasi-linear degenerate parabolic equations. Trans. Amer. Math. Soc. To appear.

[17] G.-Q. Chen and B. Perthame. Well-posedness for non-isotropic degenerate parabolichyperbolic equations. Ann. Inst. H. Poincaré Anal. Non Linéaire, 20(4):645-668, 2003.

[18] B. Cockburn and G. Gripenberg. Continuous dependence on the nonlinearities of solutions of degenerate parabolic equations. J. Differential Equations, 151(2):231-251, 1999.

[19] E. DiBenedetto. Degenerate parabolic equations. Springer-Verlag, New York, 1993.

[20] C. Ebmeyer and J. M. Urbano. Regularity in Sobolev spaces for doubly nonlinear parabolic equations. J. Differential Equations, 187(2):375-390, 2003.

[21] M. S. Espedal and K. H. Karlsen. Numerical solution of reservoir flow models based on large time step operator splitting algorithms. In Filtration in Porous Media and Industrial Applications (Cetraro, Italy, 1998), volume 1734 of Lecture Notes in Mathematics, pages 9-77. Springer, Berlin, 2000.

[22] S. Evje and K. H. Karlsen. Discrete approximations of $B V$ solutions to doubly nonlinear degenerate parabolic equations. Numer. Math., 86(3):377-417, 2000.

[23] S. Evje and K. H. Karlsen. Monotone difference approximations of $B V$ solutions to degenerate convection-diffusion equations. SIAM J. Numer. Anal., 37(6):1838-1860 (electronic), 2000.

[24] S. Evje, K. H. Karlsen, and N. H. Risebro. A continuous dependence result for nonlinear degenerate parabolic equations with spatially dependent flux function. In Hyperbolic problems: theory, numerics, applications, Vol. I (Magdeburg, 2000), pages 337-346. Birkhäuser, Basel, 2001.

[25] R. Eymard, T. Gallouët, R. Herbin, and A. Michel. Convergence of a finite volume scheme for nonlinear degenerate parabolic equations. Numer. Math., 92(1):41-82, 2002.

[26] F. Guarguaglini, V. Milisic, and A. Terracina. A discrete bgk approximation for strongly degenerate parabolic problems with boundary conditions. Preprint, 2002.

[27] N. Igbida and J. M. Urbano. Uniqueness for nonlinear degenerate problems. NoDEA Nonlinear Differential Equations Appl., 10(3):287-307, 2003.

[28] K. H. Karlsen and M. Ohlberger. A note on the uniqueness of entropy solutions of nonlinear degenerate parabolic equations. J. Math. Anal. Appl., 275(1):439-458, 2002.

[29] K. H. Karlsen and N. H. Risebro. Convergence of finite difference schemes for viscous and inviscid conservation laws with rough coefficients. M2AN Math. Model. Numer. Anal., 35(2):239-269, 2001

[30] K. H. Karlsen and N. H. Risebro. On the uniqueness and stability of entropy solutions of nonlinear degenerate parabolic equations with rough coefficients. Discrete Contin. Dyn. Syst., 9(5):1081-1104, 2003.

[31] S. N. Kružkov. First order quasi-linear equations in several independent variables. Math. USSR Sbornik, 10(2):217-243, 1970.

[32] J.-L. Lions. Quelques méthodes de résolution des problèmes aux limites non linéaires. Dunod, 1969.

[33] J. Málek, J. Nečas, M. Rokyta, and M. Ružička. Weak and measure-valued solutions to evolutionary PDEs. Chapman \& Hall, London, 1996.

[34] C. Mascia, A. Porretta, and A. Terracina. Nonhomogeneous dirichlet problems for degenerate parabolic-hyperbolic equations. Arch. Ration. Mech. Anal., 163:87-124, 2002.

[35] A. Michel and J. Vovelle. Entropy formulation for parabolic degenerate equations with general Dirichlet boundary conditions and application to the convergence of FV methods. Preprint, 2002.

[36] M. Ohlberger. A posteriori error estimates for vertex centered finite volume approximations of convection-diffusion-reaction equations. M2AN Math. Model. Numer. Anal., 35(2):355-387, 2001.

[37] F. Otto. Initial-boundary value problem for a scalar conservation law. C. R. Acad. Sci. Paris Sér. I Math., 322(8):729-734, 1996.

[38] F. Otto. $L^{1}$-contraction and uniqueness for quasilinear elliptic-parabolic equations. J. Differential Equations, 131(1):20-38, 1996.

[39] B. Perthame and P. E. Souganidis. Dissipative and entropy solutions to non-isotropic degenerate parabolic balance laws. Arch. Ration. Mech. Anal., 170(4):359-370, 2003.

[40] É. Rouvre and G. Gagneux. Solution forte entropique de lois scalaires hyperboliquesparaboliques dégénérées. C. R. Acad. Sci. Paris Sér. I Math., 329(7):599-602, 1999. 
[41] T. Tassa. Uniqueness of piecewise smooth weak solutions of multidimensional degenerate parabolic equations. J. Math. Anal. Applic., 210:598-608, 1997.

[42] J. M. Urbano. A free boundary problem with convection for the $p$-Laplacian. Rend. Mat. Appl. (7), 17(1):1-19, 1997.

[43] J. M. Urbano. A free boundary problem: contributions from modern analysis. In European Congress of Mathematics, Vol. II (Barcelona, 2000), volume 202 of Progr. Math., pages 319-329. Birkhäuser, Basel, 2001.

[44] A. I. Vol'pert. The spaces BV and quasi-linear equations. Math. USSR Sbornik, 2(2):225-267, 1967.

[45] A. I. Vol'pert and S. I. Hudjaev. Cauchy's problem for degenerate second order quasilinear parabolic equations. Math. USSR Sbornik, 7(3):365-387, 1969.

[46] J. Vovelle. Convergence of finite volume monotone schemes for scalar conservation laws on bounded domains. Numer. Math., 90:563-596, 2002.

[47] Z. Wu and J. Yin. Some properties of functions in $B V_{x}$ and their applications to the uniqueness of solutions for degenerate quasilinear parabolic equations. Northeastern Math. J., 5(4):395-422, 1989.

[48] Z. Wu, J. Zhao, J. Yin, and H. Li. Nonlinear diffusion equations. World Scientific Publishing Co. Inc., River Edge, NJ, 2001.

[49] J. Yin. On a class of quasilinear parabolic equations of second order with double-degeneracy. J. Partial Differential Equations, 3(4):49-64, 1990.

(Mostafa Bendahmane)

Instituto Gulbenkian de CiÊnCIA

Apartado 14, 2781-901 Oeiras, Portugal

E-mail address: mostafab@igc.gulbenkian.pt

(Kenneth Hvistendahl Karlsen)

Centre of Mathematics for Applications, University of Oslo

P.O. Box 1053, Blindern, N-0316 Oslo, NorWay

E-mail address: kennethk@math.uio.no

URL: http: //www . math.uio.no/ kennethk/ 